\title{
Transtibial limb loss does not increase metabolic cost in three-dimensional computer simulations of human walking
}

\author{
Ross H Miller ${ }^{\text {Corresp., 1, }}$, Elizabeth Russell Esposito ${ }^{3,4,5}$ \\ ${ }^{1}$ Department of Kinesiology, University of Maryland, College Park, Maryland, United States \\ 2 Neuroscience and Cognitive Science Program, University of Maryland, College Park, Maryland, United States \\ 3 Extremity Trauma and Amputation Center of Excellence, Fort Sam Houston, Texas, United States \\ 4 Center for Limb Loss and Mobility, Seattle, Washington, United States \\ 5 Department of Mechanical Engineering, University of Washington, Seattle, Washington, United States \\ Corresponding Author: Ross H Miller \\ Email address: rosshm@umd.edu
}

Loss of a lower limb below the knee, i.e. transtibial limb loss, and subsequently walking with a prosthesis, is generally thought to increase the metabolic cost of walking vs. ablebodied controls. However, high-functioning individuals with limb loss such as military service members often walk with the same metabolic cost as controls. Here we used a 3-D computer model and optimal control simulation approach to test the hypothesis that transtibial limb loss in and of itself causes an increase in metabolic cost of walking. We first generated $N=36$ simulations of walking at $1.45 \mathrm{~m} / \mathrm{s}$ using a "pre-limb loss" model, with two intact biological legs, that minimized deviations from able-bodied experimental walking mechanics with minimum muscular effort. We then repeated these simulations using a "post-limb loss" model, with the right leg's ankle muscles and joints replaced with a simple model of a passive transtibial prosthesis. No other changes were made to the post-limb loss model's remaining muscles or musculoskeletal parameters compared to the pre-limb loss case. Post-limb loss, the gait deviations on average increased by only 0.17 standard deviations from the experimental means, and metabolic cost did not increase $(3.58 \pm 0.10 \mathrm{~J} / \mathrm{m} / \mathrm{kg}$ pre-limb loss vs. $3.59 \pm 0.12 \mathrm{~J} / \mathrm{m} / \mathrm{kg}$ post-limb loss, $p=0.65)$. The results suggest that transtibial limb loss does not directly lead to an increase in metabolic cost, even when deviations from able-bodied gait mechanics are minimized. High metabolic costs observed in individuals with transtibial limb loss may be due to secondary changes in strength or general fitness after limb loss, modifiable prosthesis issues, or to prioritization of factors that affect locomotor control other than gait deviations and muscular effort. 


\title{
Transtibial limb loss does not increase metabolic cost in three-dimensional computer simulations of human walking
}

\author{
Ross H. Miller ${ }^{1,2, *}$ and Elizabeth Russell Esposito ${ }^{3,4,5}$ \\ ${ }^{1}$ Department of Kinesiology, University of Maryland, College Park, MD, USA \\ ${ }^{2}$ Neuroscience and Cognitive Science Program, University of Maryland, College Park, MD, USA \\ ${ }^{3}$ Extremity Trauma and Amputation Center of Excellence, Fort Sam Houston, TX, USA \\ ${ }^{4}$ Center for Limb Loss and Mobility, Seattle, WA, USA \\ ${ }^{5}$ Department of Mechanical Engineering, University of Washington, Seattle, WA, USA \\ *Corresponding author: rosshm@umd.edu
}

\begin{abstract}
Loss of a lower limb below the knee, i.e. transtibial limb loss, and subsequently walking with a prosthesis, is generally thought to increase the metabolic cost of walking vs. able-bodied controls. However, high-functioning individuals with limb loss such as military service members often walk with the same metabolic cost as controls. Here we used a 3-D computer model and optimal control simulation approach to test the hypothesis that transtibial limb loss in and of itself causes an increase in metabolic cost of walking. We first generated $N=36$ simulations of walking at $1.45 \mathrm{~m} / \mathrm{s}$ using a "pre-limb loss" model, with two intact biological legs, that minimized deviations from able-bodied experimental walking mechanics with minimum muscular effort. We then repeated these simulations using a "post-limb loss" model, with the right leg's ankle muscles and joints replaced with a simple model of a passive transtibial prosthesis. No other changes were made to the post-limb loss model's remaining muscles or musculoskeletal parameters compared to the pre-limb loss case. Post-limb loss, the gait deviations on average increased by only 0.17 standard deviations from the experimental means, and metabolic cost did not increase $(3.58 \pm 0.10 \mathrm{~J} / \mathrm{m} / \mathrm{kg}$ pre-limb loss vs. $3.59 \pm 0.12 \mathrm{~J} / \mathrm{m} / \mathrm{kg}$ post-limb loss, $p=0.65)$. The results suggest that transtibial limb loss does not directly lead to an increase in metabolic cost, even when deviations from able-bodied gait mechanics are minimized. High metabolic costs observed in individuals with transtibial limb loss may be due to secondary changes in strength or general fitness after limb loss, modifiable prosthesis issues, or to prioritization of factors that affect locomotor control other than gait deviations and muscular effort.
\end{abstract}


67

68

69

70

71

\section{INTRODUCTION}

Numerous studies report on gait deviations and a high metabolic cost of walking in individuals with lower limb amputation (e.g. Pinzur et al., 1992; Sanderson \& Martin, 1997; Rábago \& Wilken, 2016). These deviations from the mechanics and energetics of able-bodied gait are suspected to affect mobility, quality of life, and risk for chronic musculoskeletal conditions after limb loss (Molen, 1973; Gailey, 2008). Minimizing gait deviations and maintaining a reasonably economical gait are therefore important goals of rehabilitation and prosthesis prescription after limb loss. Deviations from able-bodied gait mechanics and energetics are often presumed to result directly from the amputation. However, the studies documenting these deviations are necessarily cross-sectional in nature. There are no longitudinal data on actual biomechanical and metabolic changes pre-to-post limb loss.

Longitudinal studies on gait mechanics pre- vs. post-limb loss are impractical but can be approximated in computer simulations (Zmitrewicz et al., 2007; Handford \& Srinivasan, 2016). In a previous study, motivated by observations that high-functioning children and military service members with transtibial limb loss do not have a greater metabolic cost of walking than able-bodied controls (Jeans et al., 2011; Russell Esposito et al., 2014; Jarvis et al., 2017), we used optimal control simulations to perform an in silico longitudinal study of walking mechanics and energetics pre- vs. post-limb loss. The results suggested that maintenance of residual limb muscle strength can maintain the pre-limb loss metabolic cost of walking, with only small deviations from the pre-limb loss gait mechanics (Russell Esposito \& Miller, 2018). However, the computer model in this study was limited to only the sagittal plane. Many of the reported gait deviations in individuals with limb loss occur outside the sagittal plane (Rábago \& Wilken, 2016), and lateral balance in normal human walking appears to have a modest metabolic cost (Donelan et al., 2004). Neglecting three-dimensional motion when making inferences on the energetics of walking with a prosthesis may therefore be a questionable assumption.

Therefore, the purpose of this study was to perform an in silico longitudinal study preand post-transtibial limb loss using a three-dimensional musculoskeletal model to determine the change in metabolic cost, with its typical definition as the energy expended to translate a unit mass by a unit distance (Schmidt-Nelson, 1972; Tucker, 1975). Based on previous experiments and simulations (Russell Esposito et al., 2014; Russell Esposito \& Miller, 2018), we hypothesized that the metabolic cost of walking with a transtibial prosthesis post-limb loss would be similar to the pre-limb loss metabolic cost when the pre-limb loss muscular properties and body composition were maintained.

\section{METHODS}

\subsection{Pre-Limb Loss Model}

The model was a modified version of the OpenSim (Delp et al., 2007) model described by Rajagopal et al. (2016). The pre-limb loss model is visualized in Fig. 1 and consisted of pelvis, trunk, thigh, shank, foot, toes, upper arm, and forearm rigid bodies that articulated with 31 degrees of freedom. The lumbar joint and lower limb joints were actuated by 84 total muscles (42 bilateral pairs). The shoulder and elbow joints were actuated by ideal torque generators with activation dynamics. The model had a total body mass of $75.4 \mathrm{~kg}$ and height $1.75 \mathrm{~m}$. Several changes or additions were made to the original model, which lacked some elements 
77 necessary or beneficial for optimal simulations, to make it well suited for direct collocation

78 simulations in Moco software (Dembia et al., 2020):

79

80

81

82

83

84

85

86

87

88

89

90

91

92

93

94

95

96

97

98

99

100

101

102

103

104

105

106

107

108

109

110

111

112

113

114

115

116

117

118

119

120

1. The original "Millard2012Equilibrium"-type muscles were replaced with "DeGrooteFregly2016"-type muscles, which have activation and contractile dynamics suitable for gradient-based optimization in Moco (De Groote et al., 2016).

2. Wrapping objects that defined muscle paths on the skeleton were replaced with "Conditional Path Points" that gave similar relationships between the skeletal pose and muscle lengths and moment arms. Moco does not currently support wrapping objects.

3. The studies that motivated the present study examined samples of young men (Russell Esposito et al., 2014; Jarvis et al., 2017). Therefore, muscle optimal fiber lengths and unloaded tendon lengths were adjusted so that the model the model produced maximum isometric joint torques at average joint angles for young men from Anderson et al. (2007). Specifically, optimal fiber length was adjusted unless the adjustment required shortening it to a length that prevented the muscle from producing force across a realistic range of motion, in which case unloaded tendon length was adjusted instead. The adjustments made from the original model's parameters were small, e.g. under $3 \mathrm{~cm}$ for the longest muscles in the model.

4. The original model did not include muscle-specific time constants for activation dynamics. These muscle activation dynamics time constants were defined as functions of muscle mass and fast-twitch fiber ratio using fiber-type data from Miller (2018) and time constant functions from Winters and Stark (1985).

5. The original model did not include passive forces limiting joint ranges of motion beyond the parallel elastic components of muscles. Therefore, coordinate forces in the form of passive torsional spring-dampers were added to the joints representing lumped effects of ligaments and other non-muscular joint structures on range of motion (Anderson, 1999).

Additionally, ground contact was modeled by 11 sphere-shaped Hunt-Crossley contact elements (Sherman et al., 2011) located on the plantar surface of each foot (Fig. 1). The modulus of the contact elements $(3.06 \mathrm{MPa})$ and damping coefficient $(2.0 \mathrm{~s} / \mathrm{m})$ were set so that the heel contact sphere deformation and energy return were similar to the heel region of a human foot in an athletic shoe (Aerts \& DeClercq, 1993). The frictional contact forces were a Stribeck friction model (Sherman et al., 2011) with static, dynamic, and viscous friction coefficients of $0.8,0.8$, and 0.5 , respectively, and transition velocity $0.2 \mathrm{~m} / \mathrm{s}$.

The model's muscle volumes were determined as functions of the model's mass and height using average MRI measurements of muscle volume for healthy young adults from Handsfield et al. (2014). Maximum isometric forces were the determined by dividing the volume by the muscle's optimal fiber length to determine the physiological cross-sectional area, and multiplying this area by a specific tension of $60 \mathrm{~N} / \mathrm{cm}^{2}$, the same value assumed in the original model (Rajagopal et al., 2016). This specific tension is larger than the values typically determined from in vitro single-fiber experiments, which average $\sim 13 \pm 6 \mathrm{~N} / \mathrm{cm}^{2}$ in human muscle (Rospars \& Meyer-Vernet, 2016), but is similar to the in vivo result on human quadriceps of $55 \pm 11 \mathrm{~N} / \mathrm{cm}^{2}$ for healthy adult men from O'Brien et al. (2010). The model's maximum isometric knee extension torque when emulating typical conditions of human dynamometry experiments on hip position, voluntary activation, and antagonism (Kubo et al., 
121 2006), was $3.92 \mathrm{Nm} / \mathrm{kg}$, which compares reasonably well to dynamometry experiments on

122

123

124

125

126

127

128

129

130

131

132

133

134

135

136

137

138

139

140

141

142

143

144

145

146

147

148

149

150

151

152

153

154

155

156

157

158

159

160

161

162

human subjects that reported torque-angle data (Fig. 2).

Muscle metabolic rates were calculated as functions of muscle activation and fiber velocity using the model described by Umberger et al. (2003). The muscle rates were summed and added to an assumed basal metabolic rate of $1.0 \mathrm{~W} / \mathrm{kg}$ to determine the model's wholebody metabolic rate. The metabolic rate was then averaged over time and divided by average walking speed to determine the metabolic cost, i.e. the energy expended per unit distance traveled.

\subsection{Post-Limb Loss Model}

The model of limb loss and a generic prosthesis was based on our previous 2-D simulations (Russell Esposito \& Miller, 2018). The 13 muscles spanning the right ankle were removed and the double-exponential joint torque-angle relationships representing the biological ankle, subtalar, and toe joints were replaced by linear relationships of the form:

$$
\tau=-k \theta-b \dot{\theta}
$$

where $\tau$ is the passive moment at the joint, $\theta$ is the joint angular position, and $k$ and $b$ are the stiffness and damping constants. The mass and moment of inertia of the prosthetic leg's bodies below the knee were reduced to $65 \%$ and $40 \%$, respectively, of the intact leg values, representing the typical the typical mass and mass distribution in a transtibial prosthesis (Smith \& Martin, 2013). To compute the post-limb loss model's basal metabolic rate, the minimum heat rates of all 84 pre-limb loss muscles (1.0 W per kg muscle mass; Umberger et al., 2003) were subtracted from the total basal metabolic rate $(1.0 \mathrm{~W}$ per $\mathrm{kg}$ biological mass) to determine the non-muscular basal metabolic rate ( $54 \mathrm{~W}$ average). The non-muscular rate was assumed to be unchanged post-limb loss, and the minimum heat rates of the remaining 71 post-limb muscles were added to the non-muscular rate to determine the post-limb loss basal metabolic rate. The post-limb loss model was otherwise identical to the pre-limb loss model.

A limitation of this prosthesis model is that the prosthesis was rigidly attached to the residual limb, which is more similar to an osseointegrated prosthesis than a traditional prosthesis with deformation at the socket-residuum interface. Currently, osseointegration is primarily used in patients with transfemoral limb loss who have negative outcomes using a traditional prosthesis, although transtibial osseointegration is feasible (Frossard et al., 2020). In model development, a model that allowed pistoning between the prosthesis and residual limb was tested. The force-deformation relationship of the distal residuum was a doubleexponential:

$$
F=-a_{1} \exp \left(a_{2} y\right)+a_{1} \exp \left(-2 a_{2} y\right)-b \dot{y}
$$

where $F$ is the soft tissue load at the socket-residuum interface and $y$ is the soft tissue deformation along the long axis of the residuum. Values for $a_{1}=7.8$ and $a_{2}=350$ were set to approximate the average deformation profile from $0-100 \%$ bodyweight loading from fluoroscopy data (Darter et al., 2016). Damping $b=100 \mathrm{~N} /(\mathrm{m} / \mathrm{s})$ was set to suppress highfrequency oscillations of the prosthesis during the swing phase. Adding pistoning changed the model's metabolic cost of walking by only $0.5 \%$ and did not affect its accuracy in tracking experimental gait data, so the rigid socket-residuum interface without pistoning was used for simplicity.

Peer) reviewing PDF | (2021:03:59689:2:0:NEW 12 Jul 2021) 
163

164

165

166

167

168

169

170

171

172

173

174

175

176

177

178

179

180

181

182

183

184

185

186

187

188

189

190

191

192

193

194

195

196

197

198

199

200

201

202

203

204

205

206

\subsection{Simulations}

\subsubsection{Subjects}

As a computer simulation study, the "subjects" in the present study were not specific human participants but were instances of the model described in Sections 2.1-2.2 with different parameter values. "Subject \#1" used the default values of the pre-limb loss model's parameters. To generate additional pre-limb loss subjects, a scaling factor between 0.75-1.25 was drawn randomly from a standard normal distribution and scaled all the model's maximum isometric muscle forces to adjust the model's overall strength, e.g. if the scaling factor was 1.12 , every maximum isometric force was multiplied by 1.12. Smaller random scaling factors of 0.95-1.05 that varied for each individual parameter were then applied to the scaled maximum isometric muscle forces, tendon slack lengths, and body segment masses. The body segment masses were constrained to still sum to the model's original mass and all parameter values were still required to be bilaterally symmetric, as in the original model. The ranges over which these parameters were set to produce a range of pre-limb loss metabolic costs of roughly 3.4$3.8 \mathrm{~J} / \mathrm{m} / \mathrm{kg}$. These models were then converted to post-limb loss models (Section 2.2). For each subject's post-limb loss model, a random fraction of $20-80 \%$ of the prosthetic limb's shank mass was assumed to be biological mass to represent a range of residual limb sizes.

The muscle maximum isometric forces and tendon slack lengths were perturbed because Hill model output is very sensitive to these parameter values (e.g. Scovil \& Ronsky, 2006). The segment mass distributions were perturbed because confidence in their values for a human subjects experiment is typically low. Other unmentioned parameters that could feasibly affect metabolic cost were not perturbed. The element of "subjects" in this study should essentially be viewed as a limited sensitivity analysis, hopefully providing some confidence that the results are due to the independent variable (limb loss) and not to the values assumed for parameters that are difficult to assign on a subject-specific basis with confidence. Similarly, the "subjects" here are perhaps better viewed as different parameter sets that may feasibly be assigned to an individual, or as different possible baseline fitness levels of an individual, rather than distinct individuals.

The minimum detectable effect of interest was a $2 \%$ change in metabolic cost, which is the minimum detectable change reported in testing of a modern portable pulmonary gas exchange unit due to measurement error alone, in the absence of any human biological variability between tests (Guidetti et al., 2018). The motivation for this choice of $2 \%$ is that smaller changes would be difficult to measure on human participants even if they were real changes. Simulation results from 20 subjects suggested at least 26 subjects were needed to detect the $2 \%$ effect as significant with error rates of $5 \%$ for both Type I and Type II errors. A total of 36 subjects were therefore used. In the present data, $2 \%$ equaled an effect size of 0.71 , or $\pm 0.068 \mathrm{~J} / \mathrm{m} / \mathrm{kg}$ in raw units of metabolic cost.

\subsubsection{Overview}

The models described above were used to simulate periodic strides of walking with stride duration $T=1.0 \mathrm{~s}$ and speed $1.45 \mathrm{~m} / \mathrm{s}$, the averages from healthy young adults measured by Miller et al. (2014). The simulations were posed as optimal control problems, finding the time-varying muscle excitations and associated model states that minimized a cost function $J$ that was the weighted sum of gait deviations plus muscular effort: 


$$
J=\frac{1}{T} \int_{0}^{T}\left[\frac{1}{N_{1}} \sum_{i=1}^{N_{1}}\left(\frac{x_{i}(t)-\mu_{i}(t)}{\sigma_{i}}\right)^{2}+\frac{w}{N_{2} \Delta x} \sum_{i=1}^{N_{2}} m_{i} u_{i}^{2}(t)\right] d t
$$

207 The first term on the right-hand side of Eq. 3 is the "gait deviations": $x_{i}(t)$ is the value of model

208

209

210

211

212

213

214

215

216

217

218

219

220

221

222

223

224

225

226

227

228

229

230

231

232

233

234

235

236

237

238

239

240

241

242

243

244 variable $i$ at time $t, \mu_{i}(t)$ is the mean of the analogous variable from the experimental gait analysis data of Miller et al. (2014), and $\sigma_{i}$ is the standard deviation between-subjects of the experimental data, averaged over the stride cycle. The $N_{1}=37$ variables included in this term were the six translations and rotations of the pelvis, the 25 joint angles, and the 3-D components of each ground reaction force (GRF). The square root of this term after dividing the integral by $T$ gives the average tracking error, in multiples of SD. For example, a tracking error of 1.0 indicates the model's gait mechanics were 1.0 SD away from the experimental means when averaged over all timesteps and all tracking targets. Hip internal rotation, subtalar motion, and non-sagittal shoulder motions did not track experimental data due to low confidence in accurately measuring these motions with skin marker-based motion capture. These motions tracked target angles of $0^{\circ}$ to minimize their ranges of motion, which are typically small in walking. Assigning no tracking targets for these degrees of freedom tended to produce unusual movements to track other targets unnecessarily well, e.g. the GRF. The hands were constrained to not pass through the pelvis. Lumbar joint motions were also not tracked. Instead, deviations of the trunk angle from the global axes were minimized. The standard deviations of the experimental data were still used for $\sigma_{i}$ as they were presumed to reflect the variability of the underlying motions.

The second term on the right-hand of Eq. 3 is the "muscular effort per unit distance": $u_{i}$ $(t)$ ranges from $0-1$ and is the excitation of actuator $i$ at time $t, m_{i}$ is the mass of muscle $i$, and $\Delta x$ is the horizontal displacement of the model during the stride. $N_{2}=92$ is the number of actuators in the model: 84 muscles and eight torque generators. This "muscular effort" term is a surrogate of metabolic cost and was used in the cost function instead of metabolic cost itself because (i) this version of the Moco software could not include metabolic cost in the cost function, and (ii) it is unclear if the mechanics of normal walking are consistent with a control policy that minimizes metabolic cost itself (Bertram, 2005; Ackermann \& van den Bogert, 2010). Smaller gait deviations will generally require greater muscular effort, assuming the model's minimum-effort gait is not identical to the tracking targets. The weighting constant $w$ determines the emphasis of the optimization on minimizing effort vs. deviations. Values of $w=$ 50-200/ $\bar{m}$, where $\bar{m}$ is the average muscle mass, produced realistic metabolic costs in the range of $3.0-3.8 \mathrm{~J} / \mathrm{m} / \mathrm{kg}$, typical of healthy young adults at comfortable walking speeds (Das Gupta et al., 2019), with average tracking errors typically under 1.0 SD. A value of $w=100 / \bar{m}$ was therefore used in all simulations. For the upper limb torque generators, $m_{i}$ was set equal to $\bar{m}$. The same value of $w=100 / \bar{m}$ was used in all simulations for all subjects, both pre- and postlimb loss; the value of $w$ was not further adjusted to necessarily achieve a realistic metabolic cost in every simulation, nor to minimize the change in metabolic cost with limb loss, nor any particular pre-determined result. Keeping the same value of $w$ in both the pre- and post-limb loss simulations aligns with the goal of this study in isolating the effect of limb loss.

Peer] reviewing PDF | (2021:03:59689:2:0:NEW 12 Jul 2021) 
252

253

254

255

256

257

258

259

260

261

262

263

264

265

266

267

268

269

270

271

272

273

274

275

276

277

278

279

280

281

282

283

284

285

286

287

288

The optimal control problems were converted to nonlinear programming problems and solved using a direct collocation method in Moco 0.4 .0 software (Dembia et al., 2020). Briefly, the model's state and control variables were discretized on a temporal grid of 101 nodal values spaced evenly over the stride duration. Simulation results in model development were invariant to finer grids tested up to 401 nodes per stride. These nodal values were optimized to minimize Eq. 3, subject to constraints of the skeletal equations of motion, muscle activation and contractile dynamics, and task constraints of kinematic periodicity and average speed. This method solves the same optimal control problem as the traditional forward dynamics approach in biomechanics (e.g. Neptune et al., 2001) but avoids integrating the model's state equations forward in time, which allows for high-resolution muscle excitations and relatively fast computational speeds (Van den Bogert et al., 2011). The constraint tolerance for convergence of the optimizations was $10^{-4}$.

\subsubsection{Validation simulations}

Directly validating the model's ability to predict changes in metabolic cost with limb loss is not possible since there are no longitudinal data on changes in metabolic cost pre- vs. postlimb loss. To gauge the model's validity for predicting changes in metabolic cost, we compared its predictions to literature reports on how metabolic cost changes when altering lower leg mechanics. Schertzer and Riemer (2014) added 0.5, 1.0, and $2.0 \mathrm{~kg}$ mass to each foot (weights around the ankles) and reported average respective increases in metabolic cost of $+2.9,6.7$, and $16.0 \%$. We added the same masses to our pre-limb loss model and simulation approach above, using the subject whose metabolic cost was closest to Schertzer and Riemer's (2014) mean cost with no added mass. The masses were added to the model by increasing the mass of the calcaneus segment. The cost function and simulation procedure was the same as described in Section 2.3.2. The simulated metabolic costs at all three added mass levels $(+3.5,6.5,14.7 \%)$ were within one standard deviation of the mean from the experimental data (Fig. 3a). We also evaluated the model's prediction of metabolic cost with ankle bracing. Wutzke et al. (2012) used a unilateral ankle brace that reduced ankle range of motion by $13^{\circ}$ and reported an average increase in metabolic cost of $5.8 \%$, when assuming the same resting metabolic rate as

the model. We simulated an ankle brace by adding a linear torsional spring to the model's right ankle and generating a walking simulation, again using the cost function and simulation procedure was the same as described in Section 2.3.2. With a spring stiffness of $1000 \mathrm{Nm} / \mathrm{rad}$, the ankle range of motion was reduced by $16^{\circ}$, and the metabolic cost increased by $6.2 \%$ (Fig. $3 b)$.

Changing leg mass or ankle stiffness is, of course, not the same as walking with a prosthesis, but reasonably accurate responses of the model's metabolic cost to these conditions gives some confidence in its ability to accurately predict small changes in metabolic cost for conditions that cannot easily be tested experimentally, such as pre- vs. post-limb loss.

\subsubsection{Pre-limb loss simulations}

Simulations with each subject's pre-limb loss model described in Section 2.1 were performed using the approach described in Section 2.3.2. The same tracking targets $\mu_{i}(t)$ were used in the cost function (Eq. 3) for all subjects. Each simulation was performed with three different initial guesses generated in model development and the result with the lowest cost 
289

290

291

292

293

294

295

296

297

298

299

300

301

302

303

304

305

306

307

308

309

310

311

312

313

314

315

316

317

318

319

320

321

322

323

324

325

326

327

328

329

330

function score was retained. The three "initial guess" simulations were generated using the generic pre-limb loss model with different values of $w=10 / \bar{m}, 100 / \bar{m}$, and $1000 / \bar{m}$ specified in Eq. 3. The initial guess for these simulations was the means of the tracking targets for the kinematic states and zeroes for the muscle states and controls. These simulations were first performed on a coarse collocation grid (11 nodes/stride) which helped with convergence when using a bad initial guess. The coarse result was then interpolated to generate an initial guess on a finer grid, and this process was repeated until a converged solution was achieved on the final 101-node grid.

For readers unfamiliar with the terms and methods of optimal control: (i) an "initial guess" provides a starting point for the optimization routine and contains all the variables to be optimized when generating a simulation, in this case the discretized values of the model's timevarying state and control variables; (ii) multiple initial guesses are used and the result with the lowest cost function score retained because the goal of the optimization was to minimize the cost function in an absolute sense. For example, a pre-limb loss simulation that converged on a local minimum with a high metabolic cost could bias the post-limb loss simulation towards having a comparatively low metabolic cost.

\subsubsection{Post-limb loss simulations}

Simulations with the post-limb loss models were performed identically to the pre-limb loss simulations, with the exceptions that (i) the prosthetic limb's ankle, subtalar, and toe joints were excluded from the tracking term in the cost function $\left(N_{1}=34 \mathrm{in} \mathrm{Eq.} \mathrm{3)} \mathrm{because} \mathrm{the}\right.$ tracking targets were able-bodied data, and (ii) the 13 muscles of the right ankle were removed $\left(N_{2}=79\right.$ in Eq. 3). To approximate the patient-specific prosthesis tuning process typically done in clinical practice, the stiffness and damping parameters of the three prosthetic joints (Eq. 1) were optimized in Moco for each subject's post-limb loss simulation, along with the muscle excitation controls and model states, to minimize the cost function. Stiffness was bounded on 100-1000 Nm/rad and damping on 0.01-10 Nm/(rad/s).

For all post-limb loss simulations, the same subject's pre-limb loss simulation result was used as the initial guess. The cost function in the post-limb loss simulations used the values of $\mu_{i}(t)$ from the able-bodied subjects of Miller et al. (2014) as tracking targets. The post-limb loss model was therefore not explicitly trying to track gait data from individuals with limb loss. The goal of these post-limb loss simulations was to walk with minimal deviations from a typical able-bodied gait with low muscular effort, a common goal of rehabilitation after limb loss.

\subsubsection{Outcome variable and statistics}

The outcome variable from each simulation was the gross metabolic cost, expressed as the metabolic energy consumed per unit distance traveled, divided by the biological body mass. To be clear, the pre-limb loss metabolic cost was scaled by the pre-limb loss biological mass, and the post-limb loss metabolic cost was scaled by the post-limb loss biological mass. The biological mass was $75.4 \mathrm{~kg}$ pre-limb loss and $72.8 \pm 0.3 \mathrm{~kg}$ post-limb loss. Scaling by biological body mass (excluding the prosthesis mass in the post-limb loss case) was done to avoid favoring the hypothesis of similar metabolic costs pre- vs. post-limb loss, which would have been 
331 favored by scaling the post-limb loss metabolic costs by the larger total body mass that includes

332

333

334

335

336

337

338

339

340

341

342

343

344

345

346

347

348

349

350

351

352

353

354

355

356

357

358

359

360

361

362

363

364

365

366

367

368

369

370

371

372

373

the prosthesis.

The effect of limb loss on metabolic cost was tested first with a standard Welch's $t$-test to determine if the null hypothesis of no change could be rejected. Equivalence of the pre- vs. post-limb loss metabolic costs was then tested using two one-sided tests (Lakens et al., 2018):

$$
\begin{gathered}
d \leq-d_{\text {min }} \\
d \geq d_{\text {min }}
\end{gathered}
$$

where $d$ is the effect size in the data for the post-limb loss change in metabolic cost, and $d_{\text {min }}$ is the minimum effect size of interest, defined as a $2 \%$ change earlier. When both of these hypotheses can be rejected, the change in metabolic cost lies statistically within the equivalence bounds of $-d_{\text {min }} \leq d \leq d_{\text {min }}$, suggesting the pre- vs. post-limb loss metabolic costs are practically equivalent.

\section{RESULTS}

\subsection{Gait Mechanics}

The pre-limb loss model tracked the mean experimental 3-D kinematics and GRFs with an average deviation of 0.51 SD. The post-limb loss model had an average deviation of 0.65 SD, excepting the joint angles of the prosthetic limb which were not tracked. Small non-zero GRFs appear in the "swing" phase from the foot briefly clipping the ground as the leg is swung forward.

Figures 4-7 present the time series of pre- and post-limb loss GRFs, lumbopelvic kinematics, lower limb kinematics, and upper limb kinematics, respectively, averaged over subjects. Compared to pre-limb loss, the peak anterior GRF on the prosthetic limb was reduced after limb-loss (26.7 \pm 0.5 vs. $21.2 \pm 0.6 \%$ bodyweight, $p<0.001$; Fig. 4 ), and the second peaks of the vertical GRF became bilaterally asymmetric, with on average $26 \%$ less vertical force under the prosthetic limb vs. the intact limb (106 \pm 7 vs. $132 \pm 1 \%$ bodyweight, $p<0.001$; Fig. 4$)$. Similar differences in GRFs have been reported in high-functioning individuals with transtibial limb loss vs. controls (Sanderson \& Martin, 1997). Although kinematics were not evaluated statistically, these changes in GRFs were accompanied by numerous small/subtle changes in the lumbopelvic and lower limb kinematics post-limb loss of $\sim 5^{\circ}$ of less, but few larger changes (Figs. 5 and 6). Limb loss did not affect upper limb kinematics (Fig. 7).

\subsection{Metabolic Cost}

There was no significant change in the metabolic cost of walking pre-limb loss vs. postlimb loss $(3.58 \pm 0.10 \mathrm{~J} / \mathrm{m} / \mathrm{kg}$ pre-limb loss vs. $3.59 \pm 0.12 \mathrm{~J} / \mathrm{m} / \mathrm{kg}$ post-limb loss, $p=0.65)$. The change in cost on average $(+0.006 \mathrm{~J} / \mathrm{m} / \mathrm{kg})$ fell within the equivalence bounds of $\pm 0.068 \mathrm{~J} / \mathrm{m} / \mathrm{kg}$ at the $95 \%$ confidence level $(p<0.001$; Fig. 8). Twenty of the 36 subjects $(56 \%)$ decreased metabolic cost post-limb loss. The changes in metabolic cost were generally small: of the 36 subjects, only seven (19\%) had a change outside the equivalence bounds of $\pm 0.068 \mathrm{~J} / \mathrm{m} / \mathrm{kg}$ (Fig. 9).

When the post-limb loss metabolic cost was scaled by the total system mass (including the prosthesis mass) instead of the biological body mass, the change in metabolic cost postlimb loss averaged $-0.028 \mathrm{~J} / \mathrm{m} / \mathrm{kg}$. This change was significant $(p=0.030)$ but still fell within the 
374

375

376

377

378

379

380

381

382

383

384

385

386

387

388

389

390

391

392

393

394

395

396

397

398

399

400

401

402

403

404

405

406

407

408

409

410

411

412

413

414

415

416

417

equivalence bounds (Fig. 10). When no mass scaling was performed, metabolic cost decreased post-limb loss (average $-8.8 \mathrm{~J} / \mathrm{m})$. This change was significant $(p<0.001$ ) and also outside the equivalence bounds (Fig. 10).

Table 1 presents the pre- and post-limb loss metabolic costs and their work- and heatrelated components. The right ankle muscles on average accounted for $65 \mathrm{~J}$ of metabolic energy, or $17 \%$ of the pre-limb loss metabolic cost. These muscles were absent and consumed no energy post-limb loss, but these savings were coupled with increases in energy expenditure by most other muscles post-limb loss (Fig. 11): the energy expended by the remaining 71 muscles post-limb loss exceeded their pre-limb loss energy expenditure by $52 \mathrm{~J}$ on average. The post-limb loss model still expended less total metabolic energy than the pre-limb loss model (378 J vs. $391 \mathrm{~J}$ on average), but when divided by the slightly lower biological body mass postlimb loss (72.8 kg vs. $75.4 \mathrm{~kg}$ ), the metabolic cost of transporting a unit biological mass by a unit distance did not differ pre-limb loss vs. post-limb loss.

\subsection{Prosthesis Parameters}

The optimized prosthesis stiffness parameters ( $k$ Eq. 1 ) averaged $426 \pm 51,540 \pm 63$, and $306 \pm 90 \mathrm{Nm} / \mathrm{rad}$ for the ankle, subtalar, and toe joints, respectively. The optimized damping parameters ( $b$ in Eq. 1 ) averaged $0.06 \pm 0.06,1.27 \pm 0.52$, and $0.73 \pm 0.91 \mathrm{Nm} /(\mathrm{rad} / \mathrm{s})$ for the ankle, subtalar, and toe joints, respectively. Owing to the low damping, the prosthesis had highefficiency energy return, storing an average of $27.1 \mathrm{~J}$ of mechanical strain energy and returning an average of $26.6 \mathrm{~J}$ (Table 1 ).

Figure 11 includes the energy expended by each muscle for Subject 06, who had the largest absolute increase in metabolic cost $(+0.27 \mathrm{~J} / \mathrm{m} / \mathrm{kg}$, or about $+8 \%)$. No particular muscle or small group of muscles explained this subject's increase in cost. This subject did not have outlier values for their optimized prosthesis parameters: ankle, subtalar, and toe stiffness parameters were 409,544, and $233 \mathrm{Nm} / \mathrm{rad}$, and the associated damping parameters were $0.20,1.20$, and $0.35 \mathrm{Nm} /(\mathrm{rad} / \mathrm{s})$.

\section{DISCUSSION}

The purpose of this study was to determine the change in metabolic cost when walking with a transtibial prosthesis in three-dimensional optimal control simulations. With a computer modeling approach, this study performed a pre- vs. post-limb loss comparison that is difficult to make in experiments on human subjects. Our hypothesis of similar pre- and post-limb loss metabolic costs using this three-dimensional model was supported in walking simulations. While we did not test for the effect of muscle strength as we did previously with 2-D models of limb loss (Russell Esposito \& Miller 2018), the post-limb loss model was identical to the pre-limb loss model in all parameter values including muscle strength, body mass, and mass distribution. The only difference between the pre- and post-limb loss models was the loss of the ankle muscles on the amputated side, and the replacement of the biological foot/ankle complex with a prosthetic one. This result joins a growing body of evidence suggesting that below-knee limb loss per se does not directly or inevitably cause the relatively high metabolic cost of walking seen in the general limb loss population (Jeans et al., 2011; Russell Esposito et al., 2014; Russell Esposito \& Miller, 2018). An increase in metabolic cost may instead be due to changes that 
418 tend to accompany limb loss such as loss of muscle strength or other fitness- and mobility-

419

420

421

422

423

424

425

426

427

428

429

430

431

432

433

434

435

436

437

438

439

440

441

442

443

444

445

446

447

448

449

450

451

452

453

454

455

456

457

458

459

460

461 related factors such as balance or endurance (Hewson et al., 2020).

This study was hypothesis-driven and addressed a research question with a yes/no answer, "Are metabolic costs equivalent pre- vs. post-limb loss?" The suggested mechanism for this result is that limb loss removes the metabolic cost of the lost ankle muscles but increases metabolic cost of other muscles to compensate for the lost function (Table 1; Fig. 11), with the caveat that these compensations do not necessarily need to exceed the pre-limb loss metabolic cost. The natural follow-up questions of why metabolic cost did not change on average (Fig. 8) or why some of the 36 "subjects" had different magnitudes and directions of change (Fig. 9) are interesting but are difficult to address with confidence from the present data. For example, the subject with the largest increase in metabolic cost post-limb loss did not have an obvious muscle or muscle group that appeared to explain this increase (Fig. 11), and their model parameters, prosthesis parameters, and tracking accuracy in either simulation were not unusual. Isolating the cause of this subject-specific response require additional simulations to discover why metabolic cost sometimes increased or decreased by relatively large amounts for some of the subjects, and to determine if this effect can be induced in a predictable way.

The model's tracking accuracy of human gait data (Figs. 4-7) was similar to other studies that have used this simulation method with simpler models (Van den Bogert et al., 2011; Nguyen et al., 2019) and is generally better than what can be achieved with the traditional "single shooting" approach to optimal control simulations in biomechanics (e.g. Miller et al., 2015), owing to the ability to use high-resolution muscle excitations that more closely resemble human neuromuscular control. The differences in ground reaction forces pre- vs. post-limb loss were similar to those reported in high-functioning individuals with transtibial limb loss vs. controls (Sanderson \& Martin, 1997) but the post-limb loss simulations did not exhibit the magnitude of kinematic deviations typically seen in high-functioning individuals with transtibial limb loss (Rábago \& Wilken, 2016), most of which could be reasonably expected to influence metabolic cost. As noted earlier, non-zero GRF appeared in the "swing" phase from the foot briefly clipping the ground as the leg is swung forward. These GRF were brief and small ( 50 N), are not unusual in these types of simulations (e.g. Anderson \& Pandy, 2001), and appeared in both the pre- and post-limb loss simulations, and therefore did not bias the pre- vs. post-limb loss changes in metabolic cost. Within the limitations of the present 3-D model, it was possible to walk after unilateral limb loss with a passive prosthesis without increasing metabolic cost and without major deviations from pre-limb loss gait mechanics. This result suggests that large gait deviations, even in high-functioning individuals with unilateral transtibial limb loss, may not necessarily be due to limb loss directly. Increasing the weight on muscular effort in the cost function (parameter $w$ in Eq. 3) will generally increase gait deviations and decrease metabolic cost within a subject, but the magnitude of gait deviations did not correlate strongly with metabolic cost between subjects ( $r^{2}<0.01$ for both pre- and post-limb loss). It is difficult to comment further on possible relationships between specific gait mechanics like external work and metabolic cost (e.g. Houdijk et al., 2009), as this study was not designed to determine these relationships. Relatedly, the cost function used here explicitly considered only two factors, gait deviations and muscular effort. Other unmodeled factors such as balance, comfort, and agility are also important to prosthesis users (Bell et al., 2021) and could involve trade-offs with gait deviations.

Peer] reviewing PDF | (2021:03:59689:2:0:NEW 12 Jul 2021) 
462

463

464

465

466

467

468

469

470

471

472

473

474

475

476

477

478

479

480

481

482

483

484

485

486

487

488

489

490

491

492

493

494

495

496

497

498

499

500

501

502

503

504

505

The main finding of this study of no increase in metabolic cost post-limb loss agrees with our previous conceptually similar simulation study that used a 2-D sagittal plane model (Russell Esposito \& Miller, 2018). The 3-D model expends muscular effort moving and controlling itself in the non-sagittal planes, vs. the 2-D model where these planes did not exist and all effort was expended in the sagittal plane. The 2-D model was not simply a 3-D model that only moved in the sagittal plane, it was a model for which no other planes were defined. The present 3-D result confirms that the previous 2-D result was not due to the neglected non-sagittal mechanics and gives more confidence in the generalizability of the previous result to real human walking since it was replicated here with a more complex 3-D model. We caution though that the similarity in results between these two studies with 2-D and 3-D models should not be taken to imply that the mechanics and energetics of limb loss can necessarily be modeled realistically in 2-D as a general rule. A more conservative conclusion would be that the energy cost of frontal plane balance is similar with and without transtibial limb loss. There is limited information in the limb loss literature on the energy cost of frontal plane balance. ljmker et al. (2014) reported that adding lateral stabilization via spring-cords at the waist caused small reductions in metabolic cost in individuals with transtibial limb loss (-5\%) and in controls without limb loss (-3\%), but an increase of $6.5 \%$ in individuals with transfemoral limb loss. However, these data do not necessarily imply that the cost/task of controlling balance in the frontal plane is unaffected by transtibial limb loss.

The muscle energy model includes the fraction of fast-twitch fibers as an input parameter, which affects the computed metabolic cost primarily through muscle activation/maintenance heat rate, which is a linear function of the fast-twitch fiber fraction (Umberger et al., 2003). Average values from the literature were assumed for the model's fiber-types and the same values were used for all subjects. This assumption would not be expected to have a major influence on the present results given the repeated-measures design. Our previous 2-D study used random perturbations to define fiber-types for each subject and produces the same overall finding as the present study (Russell Esposito \& Miller, 2018). However, extreme cases, such as a subject with an unusually low fraction of fast-twitch fibers in the right ankle muscles and unusually high fraction elsewhere, could feasibly produce different results than the average result reported here.

Further concerning model complexity and assumptions, the research question here was whether limb loss per se increases the metabolic cost of walking. We therefore developed a modeling approach for testing the effect of limb loss in isolation of other factors that may affect metabolic cost, such as secondary changes in fitness, a poorly adjusted prosthesis, pain during walking, etc. While the present model is on the upper end of the complexity spectrum for models used previously in these types of simulations, the model and simulation approach required simplifying assumptions compared to real humans that should be considered when generalizing the results to real human walking. For example, as noted earlier we modeled a rigid prosthesis attachment, which was justified for the sake of simplicity by the small effect of a non-rigid attachment on the primary outcome variable (metabolic cost), but the present results would not necessarily generalize to individuals who have a loose prosthesis fit or substantial discomfort at the socket-residuum interface. For another example, we did not directly minimize metabolic cost in the cost function of the simulations because this is not currently possible in the Moco software (Dembia et al., 2020) and instead used a surrogate 
506 "muscular effort" cost. This assumption is justified by the fact that the approach predicted 507 realistic magnitudes of metabolic cost and realistic changes in metabolic cost in comparison to 508 human experiments (Fig. 3), but we cannot rule out the possibility that other plausible cost 509 functions would produce different results. Lastly, the perturbations to model parameters 510 defining different "subjects" were done only to a subset of parameters. These perturbations 511 are intended to increase confidence in the effect of the independent variable (limb loss). Other 512 unperturbed parameters would surely affect metabolic cost and the variance between subjects 513 in Fig. 8, but whether they would systematically affect the change in metabolic cost with limb 514 loss in this repeated-measures design is unclear and speculative, excluding extreme cases such 515 a "subject" with ankle muscle and joint properties that are usually economical for walking.

$516 \quad$ The prosthesis stiffness and damping parameters were optimized in the post-limb loss

517 simulations to represent the clinical process of fitting/adjusting the prosthesis to the user,

518 similar to our previous 2-D study (Russell Esposito \& Miller, 2014). From a technical

519 perspective, this process also ensures that the simulated gait deviations and metabolic costs

520 are not inflated due to inadequacy in generic, unadjusted prosthesis parameters for a given

521 subject. For all subjects the optimized stiffnesses fell within the range of stiffnesses for typical

522 commercial prosthetic feet of $\sim 300-900 \mathrm{Nm} / \mathrm{rad}$ (Lehmann et al., 1993) even though bounds on

523 these parameters allowed values outside this range, lending some confidence that the

524 prosthesis model, although fairly simple, was reasonably realistic. The optimized damping

525 parameters were low, as would be expected for modern prosthetic feet with efficient

526 mechanical energy return, although some damping can theoretically be beneficial for reducing

527 damage from high-frequency vibrations (Burnett et al., 2021). The modeling approach here

528 could feasibly be adapted to subject-specific simulations for determining ideal prosthesis

529 characteristics to accomplish particular patient-specific goals, similar to proposed model-based

530 approaches on personalized gait modification and joint loading in osteoarthritis (Fregly et al.,

531 2007).

532 The prosthesis model here with its low damping had an average energy return of $98 \%$

533 (Table 1), which is rather high compared to most conventional prosthetic feet (e.g. Prince et al., 534 1998) and likely contributed to the post-limb loss metabolic costs, but is not necessarily 535 unrealistic. Recent "Pro-Flex" (Össur, Reykjavík, Iceland) prosthetic feet with carbon fiber leaf 536 springs offer considerably greater energy return than conventional feet (Childers \& Takahashi, 537 2018), in some cases appearing to approach 100\% return (Heitzmann et al., 2018), and spring538 based "energy-recycling" feet with a small $(0.8 \mathrm{~W})$ battery can also return energy at very high 539 rates (Zelik et al., 2011). However, prosthetic mechanical energy is not a direct component of 540 metabolic cost and this study was not designed for causal inferences on this rather complicated 541 relationship. For example, powering a prosthesis to act as a net generator of mechanical 542 energy does not necessarily reduce metabolic cost (Quesada et al., 2016), removing the ability 543 of the ankle to store and return energy does not necessarily increase metabolic cost

544 (Vanderpool et al., 2008), and increasing energy return does not necessarily reduce metabolic 545 cost (Zelik et al., 2011).

$546 \quad$ Metabolic cost data in biomechanics are often scaled by body mass for analysis. With 547 limb loss subjects there is a choice of whether this scaling includes or excludes the mass of the 548 prosthesis. Some studies have scaled by biological body mass excluding the prosthesis mass 549 (Russell Esposito et al., 2014), others by total mass including the prosthesis mass (Jarvis et al., 
550 2017), and others still by an unspecified mass (Jeans et al., 2011). This choice is non-trivial for 551 research questions like the present study: for a typical transtibial prosthesis mass of 1-2 kg 552 (Mattes et al., 2000), the scaling choice affects metabolic cost by $1-3 \%$ for body masses 553 ranging from $70-100 \mathrm{~kg}$, which can feasibly change conclusions when the minimum effect of 554 interest is $\sim 2 \%$. Here regardless of the choice of scaling, limb loss did not increase metabolic 555 cost, but different conclusions could be reached depending on the scaling choice (Fig. 10). With

556

557

558

559

560

561

562

563

564

565

566

567

568

569

570

571

572

573

574

575

576

577

578

579

580

581

582

583

584

585

586

587

588

589

590

591

592

593 no scaling, limb loss decreased metabolic cost, and the decrease was greater than the $2 \%$ minimum change of interest. With total mass scaling, inclusive of both the biological mass and the prosthesis mass, limb loss also decreased metabolic cost, but the decrease was less than the defined meaningful difference of $2 \%$. With biological mass scaling only, limb loss did not change the metabolic cost; the average result was a small increase in cost but this increase was too small to be meaningful. We contend that to address our specific research question on whether limb loss per se changes metabolic cost, scaling by biological mass is the most appropriate and conservative choice since it accounts for change in biologic tissue mass that occurs with limb loss, and divides the energy consumed by a smaller mass than the total mass to avoid favoring the hypothesis of no increase in cost post-limb loss. Metabolic cost scaled by biological mass could be interested as a metric of efficiency, the energy expended to walk by the available biological mass that is capable of consuming energy, while scaling by total mass could be interested as a metric of economy, the energy expended to transport a unit mass. Regardless of this choice, many studies on limb loss that involved mass scaling do not report the specific mass that outcome variables were scaled by. There are certainly cases where scaling by total mass rather than biological mass is a sensible approach. We are not advocating that limb loss data should always be scaled by biological mass regardless of the research question. However, reporting the specific mass used for scaling is critical for interpreting metabolic energy expenditure data and should be mandatory.

The choice of a $2 \%$ change in metabolic cost to define the minimum "meaningful" effect of interest was based on a recent study on the reliability of modern portable metabolic units (Guidetti et al., 2018). The smallest "clinically relevant" change for walking with limb loss or for walking in general is not well defined to our knowledge, but is presumably at least as large as the minimum reliably measurable difference used here. The small value used here avoided favoring our hypothesis of no necessary increase in metabolic cost with limb loss. In addition, "small" changes in gross metabolic cost (under 5-10\%) are often of interest in prosthesis research (e.g. Zelik et al., 2011; Nolan, 2012).

A final limitation of note is that these results pertain to unilateral transtibial limb loss only, despite any ambiguous references in this report to general "limb loss". We would expect similar results for simulations of lower-level limb loss, e.g. mid-foot, but not for higher-level limb loss e.g. above-knee or bilateral limb loss, but these expectations are speculative. Relatedly, these results do not necessarily generalize to other more demanding movements such as running or walking with substantial load carriage.

In conclusion, walking with a transtibial prosthesis after below-knee limb loss did not increase the metabolic cost in optimal control simulations with a 3-D musculoskeletal model. This finding supports our previous similar finding from a simpler 2-D model. The results further suggest that major deviations in the body's intact joints and segments from able-bodied gait are also not an inevitable consequence of limb loss and may be due to secondary changes in 
594 fitness, or to priorities in the motor control of gait other than muscular effort. Lastly, studies on

595 limb loss should report the mass used in scaling outcome variables.

596

597

598

599

600

601

602

603

604

605

606

607

608

609

610

611

612

613

614

615

616

617

\section{References}

Ackermann M, van den Bogert AJ (2010). Optimality principles for model-based prediction of human gait. Journal of Biomechanics 43, 1055-1060. DOI: 10.1016/j.jbiomech.2009.12.012

Aerts P, De Clercq D (1993). Deformation characteristics of the heel region of the shod foot during a simulated heel strike: the effect of varying midsole hardness. Journal of Sports Sciences 11, 449-461. DOI: 10.1080/02640419308730011

Anderson DE, Madigan ML, Nussbaum MA (2007). Maximum voluntary joint torque as a function of joint angle and angular velocity: model development and application to the lower limb. Journal of Biomechanics 40, 3105-3113. DOI: 10.1016/j.jbiomech.2007.03.022

Anderson FC, Pandy MG (2001). Dynamic optimization of human walking. Journal of Biomechanical Engineering 123, 381-390. DOI: 10.1115/1.1392310

Bell EM, Carrignan J, Collier DK, Yang JS, Shim JK (2021). Identifying prosthetic capabilities that define functionality of individuals with amputation. Journal of Prosthetics \& Orthotics. DOI: 10.1097/JPO.0000000000000356

Bertram JEA (2005). Constrained optimization in human walking: cost minimization and gait plasticity. Journal of Experimental Biology 208, 979-991. DOI: 10.1242/jeb.01498

Burnett JK, Choi YT, Li H, Wereley NM, Miller RH, Shim JK (2021). Vibration suppression of a composite prosthetic foot using piezoelectric shunt damping: implication to vibration-induced cumulative trauma. IEEE Transactions on Biomedical Engineering. DOI: 10.1109/TBME.2021.3053374

Childers WL, Takahashi KZ (2018). Increasing prosthetic foot energy return affects whole-body mechanics during walking on level ground and slopes. Scientific Reports 8, article 5354. DOI: 10.1038/s41598-018-23705-8

619

620

621

622

623

Darter BJ, Sinitski K, Wilken JM (2016). Axial bone-socket displacement for persons with a traumatic transtibial amputation: the effect of elevated vacuum suspension at progressive body-weight loads. Prosthetics \& Orthotics International 40, 552-557. DOI: 10.1177/0309364615605372

624

625

626

Das Gupta, Bobbert MF, Kistemaker DA (2019). The metabolic cost of walking in healthy young and older adults: a systematic review and meta analysis. Scientific Reports 9, 9956. DOI: 10.1038/s41598-019-45602-4

627

628

629

De Groote F, Kinney AL, Rao AV, Fregly BJ (2016). Evaluation of direct collocation optimal control problem formulations for solving the muscle redundancy problem. Annals of

630

631 Biomedical Engineering 44, 2922-2936. DOI: 10.1007/s10439-016-1591-9

De Ruiter CJ, Kooistra RD, Paalman MI, de Haan A (2004). Initial phase of maximal voluntary and electrically stimulated knee extension torque development at different knee angles. Journal of Applied Physiology 97, 1693-1701. DOI: 10.1151/japplphysiol.00230.2004

634 Delp SL, Anderson FC, Arnold AS, Loan P, Habib A, John CT, Guendelman E, Thelen DG (2007). OpenSim: open-source software to create and analyze dynamic simulations of movement.

636 IEEE Transactions on Biomedical Engineering 54, 1940-1950. DOI: 10.1109/TBME.2007.901024 
637 Dembia CL, Bianco NA, Falisse A, Hicks JL, Delp SL (2020). OpenSim Moco: musculoskeletal

638

639

640

641

642

643

644

645

646

647

648

649

650

651

652

653

654

655

656

657

658

659

660

661

662

663

664

665

666

667

668

669

670

671

672

673

674

675

676

677

678

679 optimal control. PLoS Computational Biology 16, e1008493. DOI:

10.1371/journal.pcbi.1008493

Donelan JM, Shipman DW, Kram R, Kuo AD (2004). Mechanical and metabolic requirements for active lateral stabilization in human walking. Journal of Biomechanics 37, 827-835. DOI: 10.1016/j.jbiomech.2003.06.002

Fregly BJ, Reinbolt JA, Rooney KL, Mitchell KH, Chmielewski TL (2007). Design of patient-specific gait modifications for knee osteoarthritis rehabilitation. IEEE Transactions on Biomedical Engineering 54, 1687-1695. DOI: 10.1109/tbme.2007.891934

Frey-Law LA, Laake A, Avin KG, Heitsman J, Marler T, Abdel-Malek K (2012). Knee and elbow 3D strength surfaces: peak torque-angle-velocity relationships. Journal of Applied Biomechanics 28, 726-737. DOI: 10.1123/jab.28.6.726

Frossard L, Leech B, Pitkin M (2020). Loading applied on osseointegrated implant by transtibial bone-anchored prostheses during daily activities: preliminary characterization of prosthetic feet. Journal of Prosthetics \& Orthotics 32, 258-271. DOI: 10.1097/JPO.0000000000000280

Gailey R, Allen K, Castles J, Kucharik J, Roeder M (2008). Review of secondary physical conditions associated with lower-limb amputation and long-term prosthesis use. Journal of Rehabilitation Research \& Development 45, 15-30. DOI: 10.1682/JRRD.2006.11.0147 Guidetti L, Meucci M, Bolletta F, Emerenziani GP, Gallotta MC, Baldari C (2018). Validity, reliability and minimum detectable change of COSMED K5 portable gas exchange system in breath-by-breath mode. PLoS One 13, e0209925. DOI: 10.1371/journal. pone.0209925 Handford ML, Srinivasan M (2016). Robotic lower limb prosthesis design through simultaneous computer optimizations of human and prosthesis costs. Scientific Reports 6, 19983. DOI: 10.1038/srep19983

Handsfield GG, Meyer CH, Hart JM, Abel MF, Blemker SS (2014). Relationships of 35 lower limb muscles to height and body mass quantified using MRI. Journal of Biomechanics 47, 631-638. DOI: 10.1016/j.jbiomech.2013.12.002

Heitzmann DWW, Salami F, De Asha AR, Block J, Putz C, Wolf SI, Alimusaj M (2018). Benefits of an increased prosthetic ankle range of motion for individuals with a trans-tibial amputation walking with a new prosthetic foot. Gait \& Posture 64, 174-180. DOI: 10.1016/j.gaitpost.2018.06.022

Hewson A, Dent S, Sawers A (2020). Strength deficits in lower limb prosthesis users: a scoping review. Prosthetics \& Orthotics International 44, 323-340. DOI: 10.1177/0309364620930176 Houdijk H, Pollmann E, Groenewold M, Wiggerts H, Polomski W (2009). The energy cost for step-to-step transitions in amputee walking. Gait \& Posture 30, 35-40. DOI: 10.1016/j.gaitpost.2009.02.09

ljmker T, Noten S, Lamoth CJ, Beek PJ, van der Woude LHV, Houdijk H (2014). Can external lateral stabilization reduce the energy cost of walking in persons with a lower limb amputation? Gait \& Posture 40, 616-621. DOI: 10.1016/j.gaitpost.2014.07.013 Jarvis HL, Bennett AN, Twiste M, Phillip RD, Etherington J, Baker R (2017). Temporal spatial and metabolic measures of walking in highly functional individuals with lower limb amputations 10.1016/j.apmr.2016.09.134

Peer] reviewing PDF | (2021:03:59689:2:0:NEW 12 Jul 2021) 
680 Jeans KA, Browne RH, Karol LA (2011). Effect of amputation level on energy expenditure during overground walking by children with an amputation. Journal of Bone \& Joint Surgery 93A, 49-

682 56. DOI: 10.2106/JBJS.I.01557

683 Kubo K, Tsunoda N, Kanehisa H, Fukunaga T (2004). Activation of agonist and antagonist muscles at different joint angles during maximal isometric efforts. European Journal of Applied Physiology 91, 349-352. DOI: 10.1007/s00421-003-1025-x

686

Kubo K, Ohgo K, Takeishi R, Yoshinaga K, Tsunoda N, Kanehisa H, Fukunaga T (2006). Effects of

687

688 series elasticity on the human knee extension torque-angle relationship in vivo. Research

689 Quarterly for Exercise \& Sport 77, 408-416. DOI: 10.1080/02701367.2006.10599376

690

691

Lakens D, Schell AM, Isager PM (2018). Equivalence testing for psychological research: a tutorial. Advances in Methods \& Practices in Psychological Science 1, 259-269. DOI: $10.1177 / 2515245918770963$

Lanza MB, Balshaw TG, Folland JP (2019). Is the joint-angle specificity of isometric resistance training real? And if so, does it have a neural basis? European Journal of Applied Physiology 119, 2465-2476. DOI: 10.1007/s00421-019-04229-z

Lehmann JF, Price R, Boswell-Bessette S, Dralle A, Questad K (1993). Comprehensive analysis of dynamic elastic response feet: Seattle ankle/lite foot versus SACH foot. Archives of Physical Medicine \& Rehabilitation 74, 853-861. DOI: 10.1016/0003-9993(93)90013-z unilateral transtibial amputations: matching prosthetic and intact limb inertial properties. Archives of Physical Medicine \& Rehabilitation 81, P561-P568. DOI: 10.1016/s0003-

701

702

703 9993(00)90035-2

Miller RH, Edwards WB, Brandon SCE, Morton AM, Deluzio KJ (2014). Why don't most runners get knee osteoarthritis? A case for per-unit-distance loads. Medicine \& Science in Sports \&

704

705 Exercise 46, 572-579. DOI: 10.1249/MSS.0000000000000135

Molen NH (1973). Energy/speed relation of below-knee amputees walking on a motor-driven treadmill. Internationale Zeitschrift für Angewandte Physiologie einschließlich Arbeitsphysiologie 31, 173-185. DOI: 10.1007/BF00697597 support, forward progression and swing initiation during walking. Journal of Biomechanics 34, 1387-1398. DOI: 10.1016/S0021-9290(01)00105-1

Nguyen VQ, LaPre AK, Price MA, Umberger BR, Sup FC (2020). Inclusion of actuator dynamics in simulations of assisted human movement. International Journal for Numerical Methods in

713

714

715

716

717

718

719

720

721 Biomedical Engineering 36, e3334. DOI: 10.1002/cnm.3334

Nolan L (2012). A training programme to improve in persons with lower limb amputation. Journal of Rehabilitation Medicine 44, 241-248. DOI: 10.2340/16501977-0921

O'Brien TD, Reeves ND, Baltzopoulos V, Jones DA, Maganaris CN (2009). The effects of agonist and antagonist muscle activation on the knee extension moment-angle relationship in adults and children. European Journal of Applied Physiology 106, 849-856. DOI: 10.1007/s00421009-1088-4

O'Brien TD, Reeves ND, Baltzopoulos V, Jones DA, Maganaris CN (2010). In vivo measurements of muscle specific tension in adults and children. Experimental Physiology 95, 202-210. DOI:

722 10.1113/expphysiol.2009.048967 
723 Pincivero DM, Salfetnikov Y, Campy RM, Coelho AJ (2004). Angle- and gender-specific

724 quadriceps femoris muscle recruitment and knee extensor torque. Journal of Biomechanics

725 37, 1689-1697. DOI: 10.1016/j.jbiomech.2004.02.005

726 Pinzur MS, Gold J, Schwartz D, Gross N (1992). Energy demands for walking in dysvascular

727

728

729

730

731

732

733

734

735

736

737

738

739

740

741

742

743

744

745

746

747

748

749

750

751

752

753

754

755

756

757

758

759

760

761

762

763

764 amputees as related to level of amputation. Orthopaedics 15, 1033-1036. DOI: 10.3928/0147-7447-19920901-07

Prince F, Winter DA, Sjonnensen G, Powell C, Wheeldon RK (1998). Mechanical efficiency during gait of adults with transtibial amputation: a pilot study comparing the $\mathrm{SACH}$, Seattle, and Golden-Ankle prosthetic feet. Journal of Rehabilitation Research \& Development 35, 177185. No DOI available.

Quesada RE, Caputo MJ, Collins SH (2016). Increasing ankle push-off work with a powered prosthesis does not necessarily reduce metabolic rate for transtibial amputees. Journal of Biomechanics 49, 3452-3459. DOI: 10.1016/j.jbiomech.2016.09.015

Rábago CA, Wilken JM (2016). The prevalence of gait deviations in individuals with transtibial amputation. Military Medicine 181, 30-37. DOI: 10.7205/MILMED-D-15-00505

Rajagopal A, Dembia CL, Demers MS, Delp DD, Hicks JL, Delp SL (2016). Full-body musculoskeletal model for muscle-driven simulation of human gait. IEEE Transactions on Biomedical Engineering 63, 2068-2079. DOI: 10.1109/TBME.2016.2586891

Rospars JP, Meyer-Vernet N (2016). Force per cross-sectional area from molecules to muscles: a general property of biological motors. Royal Society Open Science 3, 160313. DOI: 10.1098/rsos.160313

Russell Esposito E, Rodriguez KM, Rábago CA, Wilken JM (2014). Does unilateral transtibial amputation lead to greater metabolic demand during walking? Journal of Rehabilitation Research \& Development 51, 1287-1296. DOI: 10.1682/JRRD.2014.06.0141

Russell Esposito E, Miller RH (2018). Maintenance of muscle strength retains a normal metabolic cost in simulated walking after transtibial limb loss. PLoS One 13, e0191310. DOI: 10.1371/journal.pone.0191310

Sanderson DJ, Martin PE (1997). Lower extremity kinematic and kinetic adaptations in unilateral below-knee amputees during walking. Gait \& Posture 6, 126-136. DOI: 10.1016/S0966-6362(97)01112-0

Schertzer E, Riemer R (2014). Metabolic rate of carrying added mass: A function of walking speed, carried mass and mass location. Applied Ergonomics 45, 1422-1432. DOI: 10.1016/j.apergo.2014.04.009

Schmidt-Nelson K (1972). Locomotion: energy cost of swimming, flying, and running. Science 177, 222-228. DOI: $10.1126 /$ science.177.4045.222

Sherman MA, Seth AJ, Delp SL (2011). Simbody: multibody dynamics for biomedical research. Procedia IUTAM 2, 241-261. DOI: 10.1016/j.piutam.2011.04.023

Smith JD, Martin PE (2013). Effects of prosthetic mass distribution on metabolic costs and walking symmetry. Journal of Applied Biomechanics 29, 317-328. DOI: 10.1123/jab.29.3.317 Tucker TA (1975). The energetic cost of moving about: walking and running are extremely inefficient forms of locomotion. Much greater efficiency is achieved by birds, fish, and bicyclists. American Scientist 63, 413-419. No DOI available. 
765

766

767

768

769

770

771

772

773

774

775

776

777

778

779

780

781

782

783

784

785

786

787
Umberger BR, Gerritsen KGM, Martin PE (2003). A model of human muscle energy expenditure. Computer Methods in Biomechanics \& Biomedical Engineering 6, 99-111. DOI: 10.1080/1025584031000091678

Van den Bogert AJ, Blana D, Heinrich D (2011). Implicit methods for efficient musculoskeletal simulation and optimal control. Procedia IUTAM 2, 297-316. DOI: 10.1016/j.piutam.2011.04.027

Vanderpool MT, Collins SH, Kuo AD (2008). Ankle fixation need not increase the energetic cost of human walking. Gait \& Posture 28, 427-433. DOI: 10.1016/j.gaitpost.2008.01.016

Winters JM, Stark L (1985). Analysis of fundamental human movement patterns through the use of in-depth antagonistic muscle models. IEEE Transactions on Biomedical Engineering 32, 826-839. DOI: 10.1109/TBME.1985.325498

Wutzke CJ, Sawicki GS, Lewek MD (2012). The influence of a unilateral fixed ankle on metabolic and mechanical demands during walking in unimpaired young adults. Journal of

Biomechanics 45, 2405-2410. DOI: 10.1016/j.jbiomech.2012.06.035

Zelik KE, Collins SH, Adamczyk PG, Segal AD, Klute GK, Morgenroth DC, Hahn ME, Orendurff MS, Czerniecki JM, Kuo AD (2011). Systematic variation of prosthetic foot spring affects center-ofmass mechanics and metabolic cost during walking. IEEE Transactions on Neural Systems \& Rehabilitation Engineering 19, 411-419. DOI: 10.1109/TNSRE.2011.2159018

Zmitrewicz RJ, Neptune RR, Sasaki K (2007). Mechanical energetic contributions from individual muscles and elastic prosthetic feet during symmetric unilateral transtibial amputee walking: a theoretical study. Journal of Biomechanics 40, 1824-1831. DOI: 10.1016/j.jbiomech.2006.07.009 
Figure 1

Graphic of the OpenSim model

Figure 1: The pre-limb loss model visualized in OpenSim software. (a) Full body with all joint angles set to zero. (b) Plantar surface of the foot with contact spheres. 
(a)

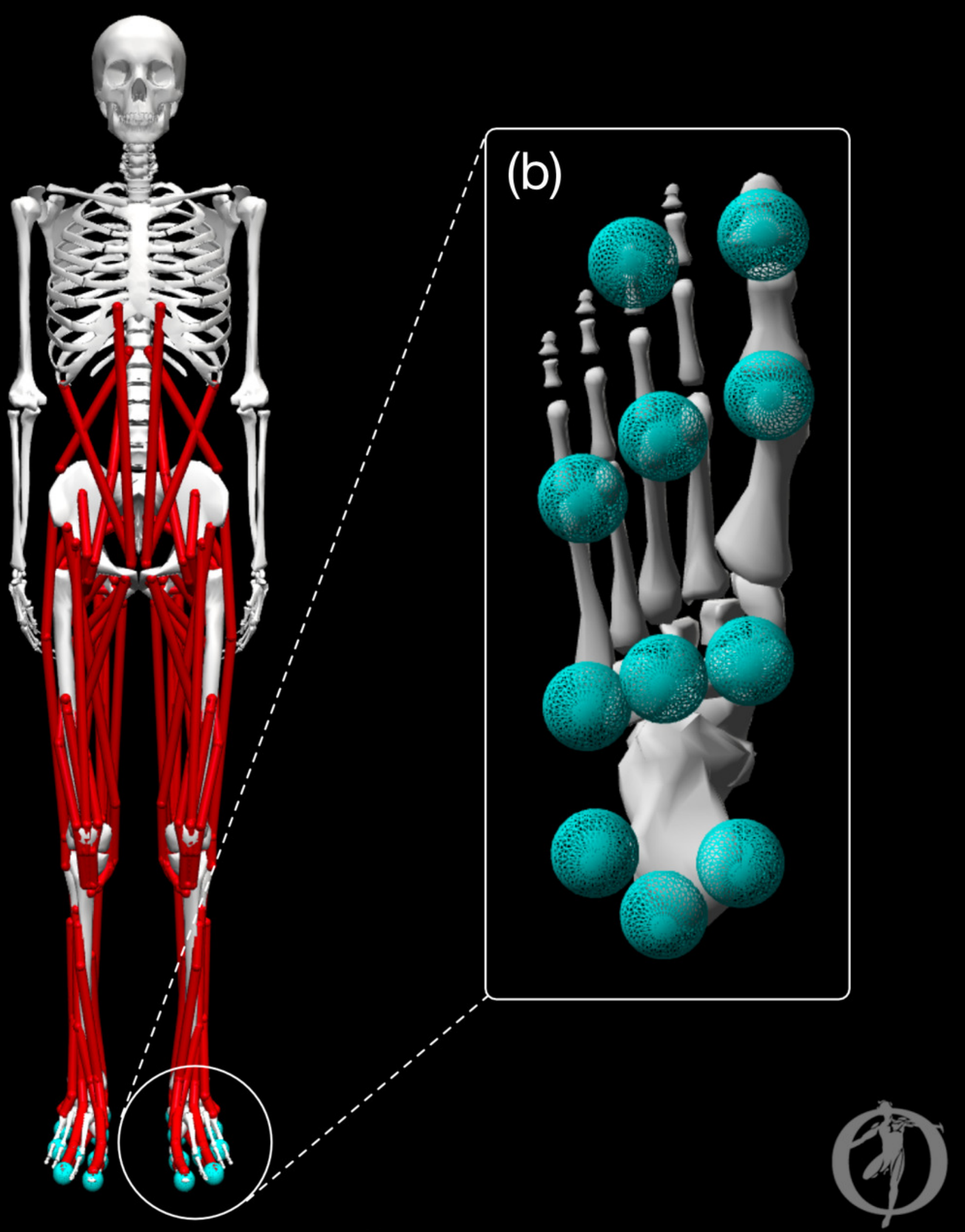




\section{Figure 2}

Maximum isometric knee extension torque of the model

Figure 2: The model's maximum isometric knee extension torque when simulating typical conditions from human dynamometry tests for hip position, agonist activation, and antagonist activation (Kubo et al., 2004). Circles are means with standard deviation bars for similar dynamometry experiments on healthy young men. Data from Lanza et al. (2019) are before and after four weeks of quadriceps strength training.

Lanza et al. (2019), post-training Lanza et al. (2019), pre-training

Kubo et al. (2006) de Ruiter et al. (2004) Pincivero et al. (2004) O'Brien et al. (2009) Anderson et al. (2007) Frey-Law et al. (2012)

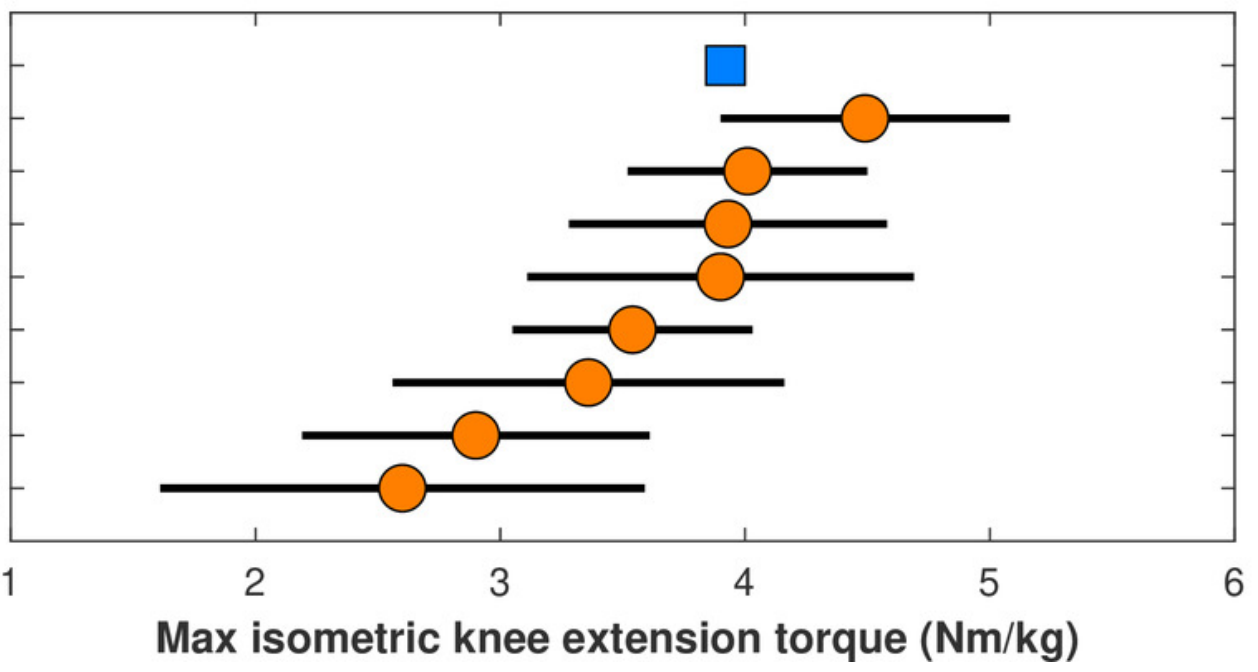


Figure 3

Gauging validity of model's metabolic cost predictions vs. human experimental results

Figure 3: Metabolic costs of simulations with (a) added foot mass or (b) an ankle brace, compared to human experimental data that tested similar conditions. Error bars are one standard deviation around the experimental mean.

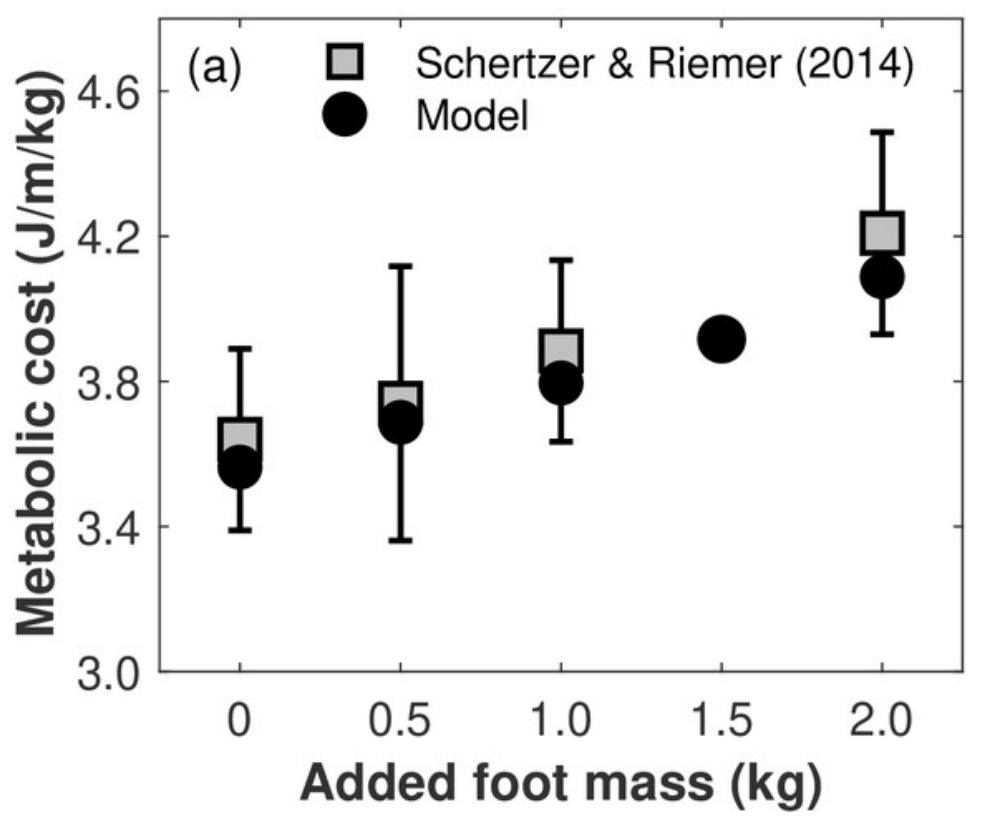

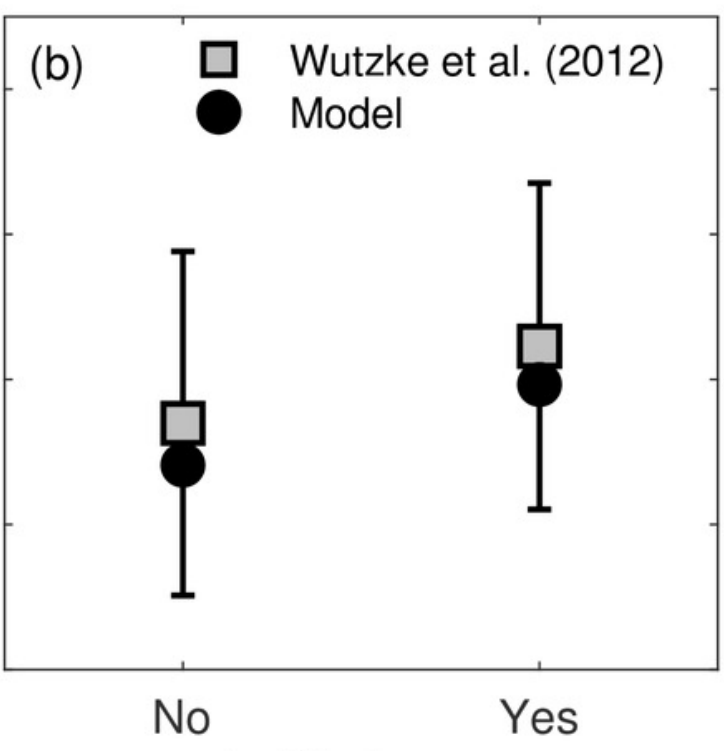

Ankle brace 
Figure 4

Simulated ground reaction forces

Figure 4: Ground reaction forces (GRF) in the vertical, anterior-posterior (AP), and mediallateral (ML) directions during the stride cycle for the pre-limb loss (solid lines) and post-limb loss (broken lines) simulations. Data begin and end at heel-strike of the leg indicated in the column headings. Shaded areas are \pm one standard deviation around the mean of instrumented gait analysis measurements from humans walking at the same average speed (Miller et al., 2014).
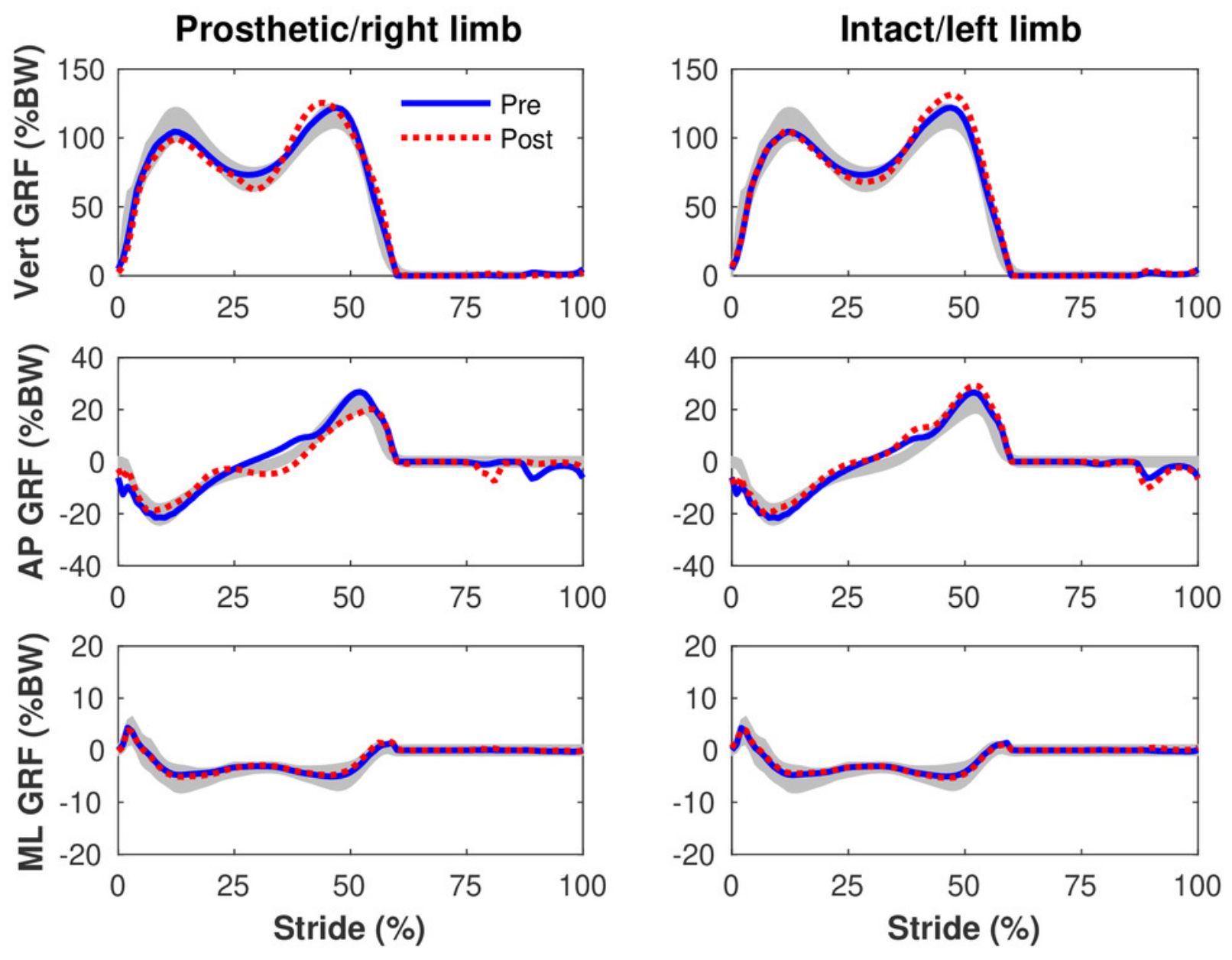
Figure 5

Simulated lumbo-pelvic kinematics

Figure 5: Pelvis and lumbar joint angles during the stride cycle for the pre-limb loss (solid lines) and post-limb loss (broken lines) simulations. Data begin and end at heel-strike of the right leg, the prosthetic leg post-limb loss. Shaded areas are \pm one standard deviation around the mean of instrumented gait analysis measurements from humans walking at the same average speed (Miller et al., 2014).
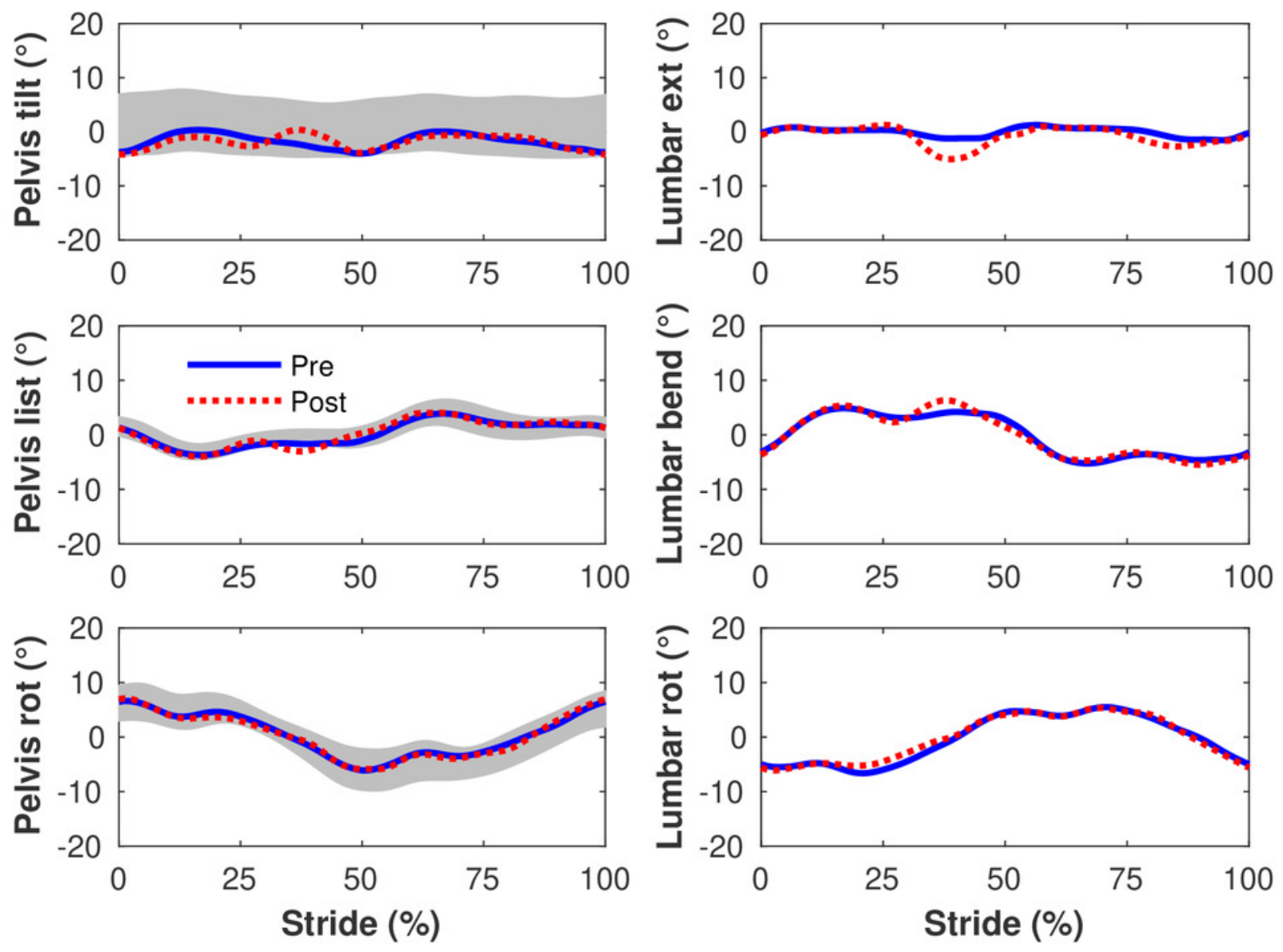


\section{Figure 6}

Simulated lower limb kinematics

Figure 6: Lower limb joint angles during the stride cycle for the pre-limb loss (solid lines) and post-limb loss (broken lines) simulations. Data begin and end at heel-strike of the leg indicated in the column headings. Shaded areas are \pm one standard deviation around the mean of instrumented gait analysis measurements from humans walking at the same average speed (Miller et al., 2014). 

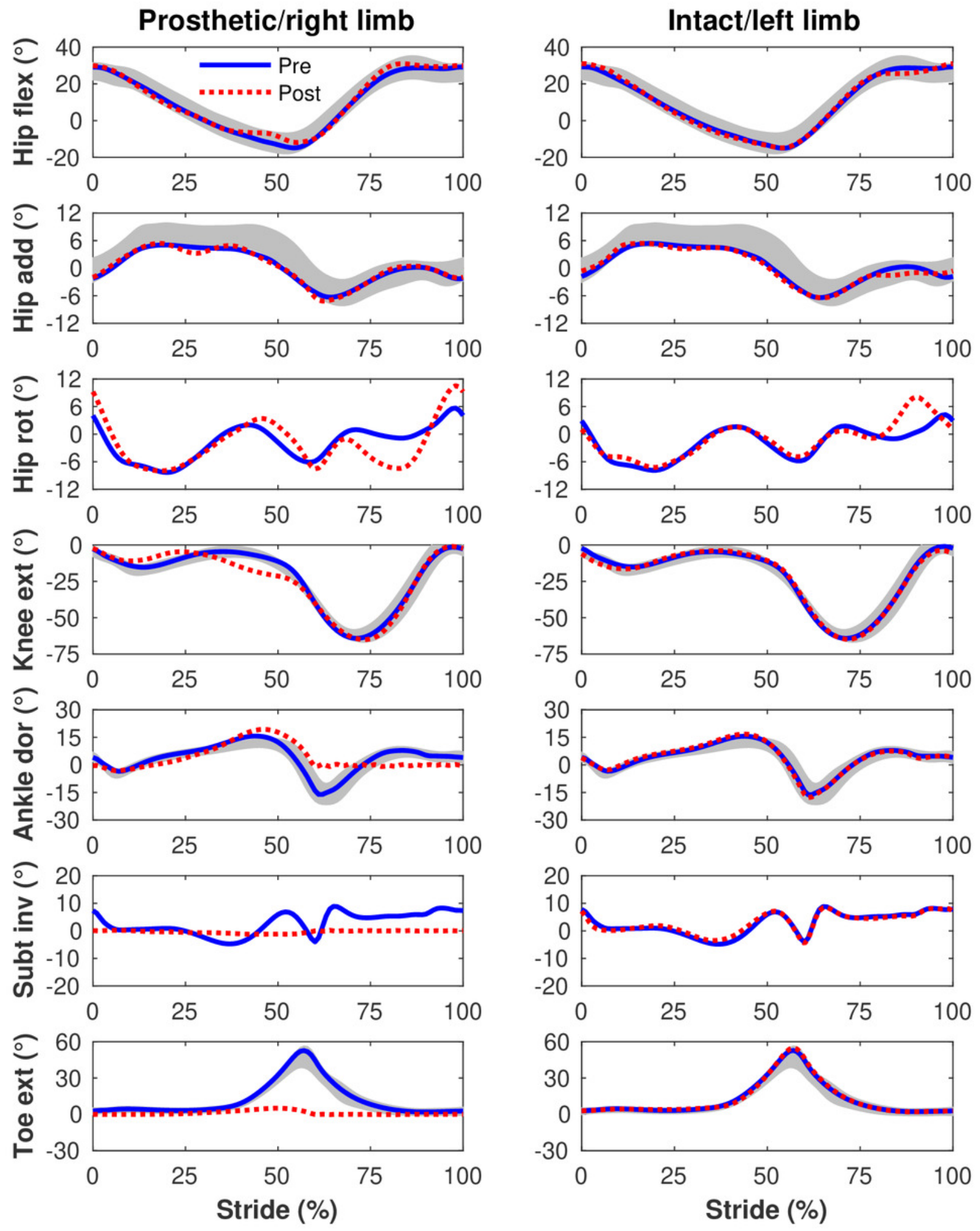


\section{Figure 7}

Simulated upper limb kinematics

Figure 7: Upper limb joint angles during the stride cycle for the pre-limb loss (solid lines) and post-limb loss (broken lines) simulations. Data begin and end at heel-strike of the leg indicated in the column headings. Shaded areas are \pm one standard deviation around the mean of instrumented gait analysis measurements from humans walking at the same average speed (Miller et al., 2014). 

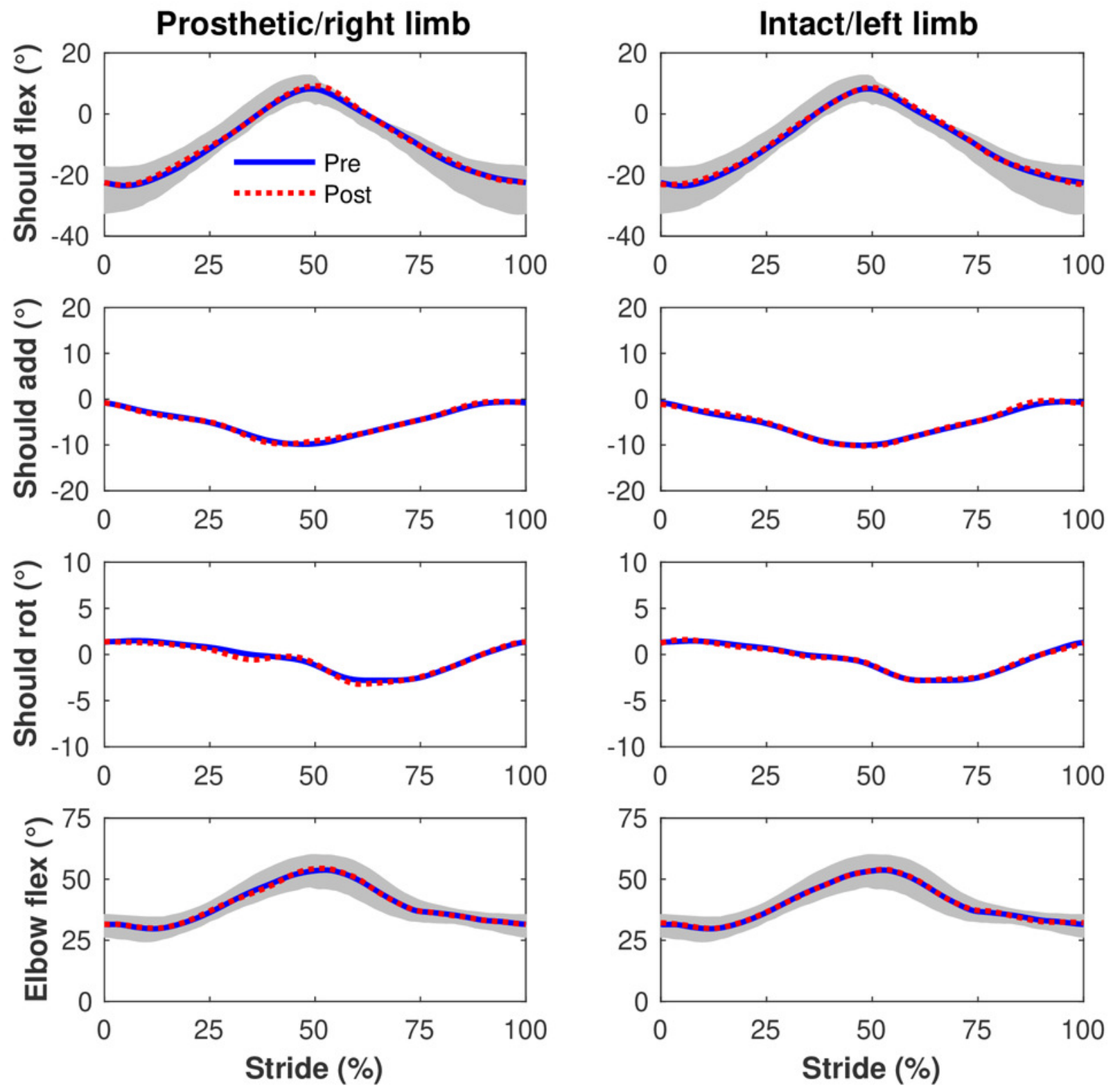
Figure 8

Mean change in metabolic cost with confidence intervals for TOST procedure

Figure 8: Mean change in metabolic cost from pre- to post-limb loss, with $90 \%$ and $95 \%$ confidence intervals. The change is was not significantly different from zero since the $95 \%$ confidence interval included zero, and was significantly less than the minimum effect of interest since the $90 \%$ confidence interval did not intercept either equivalence bound.

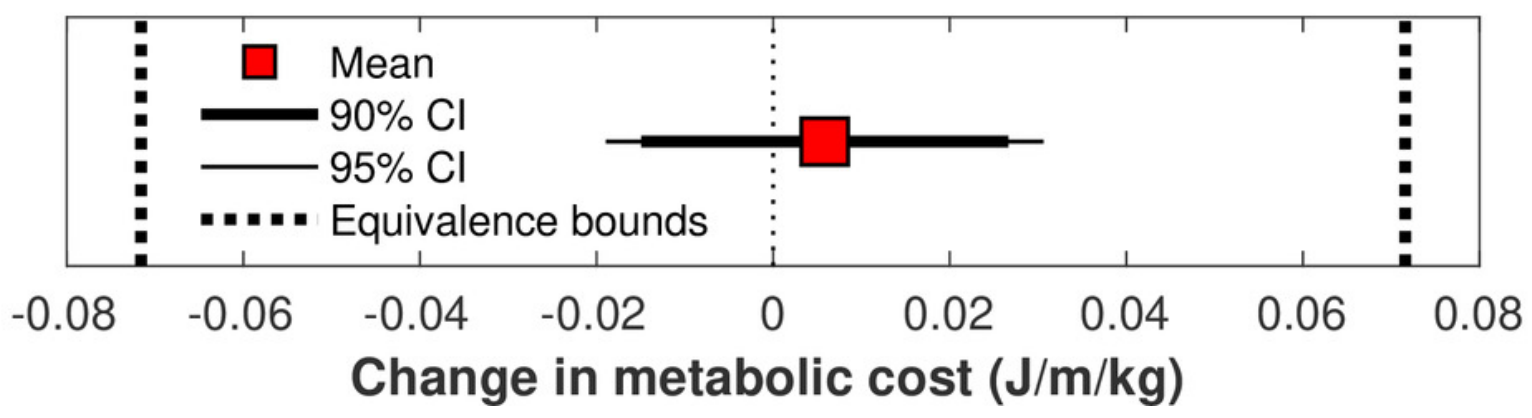


Figure 9

Subject-specific metabolic costs

Figure 9: Metabolic costs for each of the 36 subjects, pre- and post-limb loss. Symbol pairs with closed circles and solid lines had an increased cost post-limb loss. Symbol pairs with open circles and broken lines had a decreased cost post-limb loss. The larger square symbols are mean costs for each condition. 


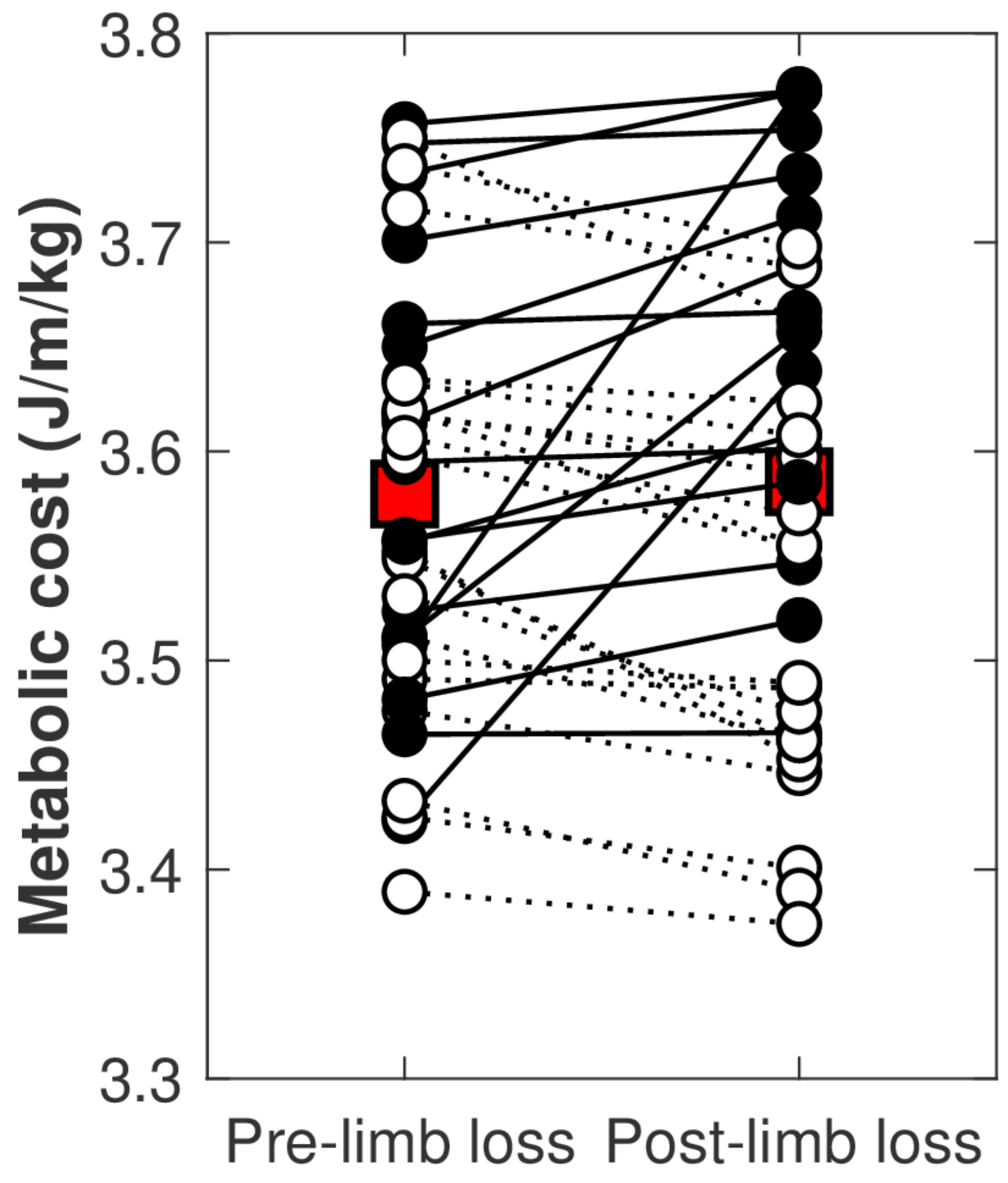




\section{Figure 10}

Effect of body mass scaling on change in metabolic cost

Figure 10: Effect sizes for mean change in metabolic cost from pre- to post-limb loss if the post-limb loss metabolic cost was scaled by the biological body mass (excluding the prosthesis), by the total body mass (including the prosthesis), or unscaled by any mass. A change is significantly different from zero if the $95 \%$ confidence interval does not cross zero, and is significantly less than the minimum effect of interest is the $90 \%$ confidence interval does not intercept either equivalence bound.

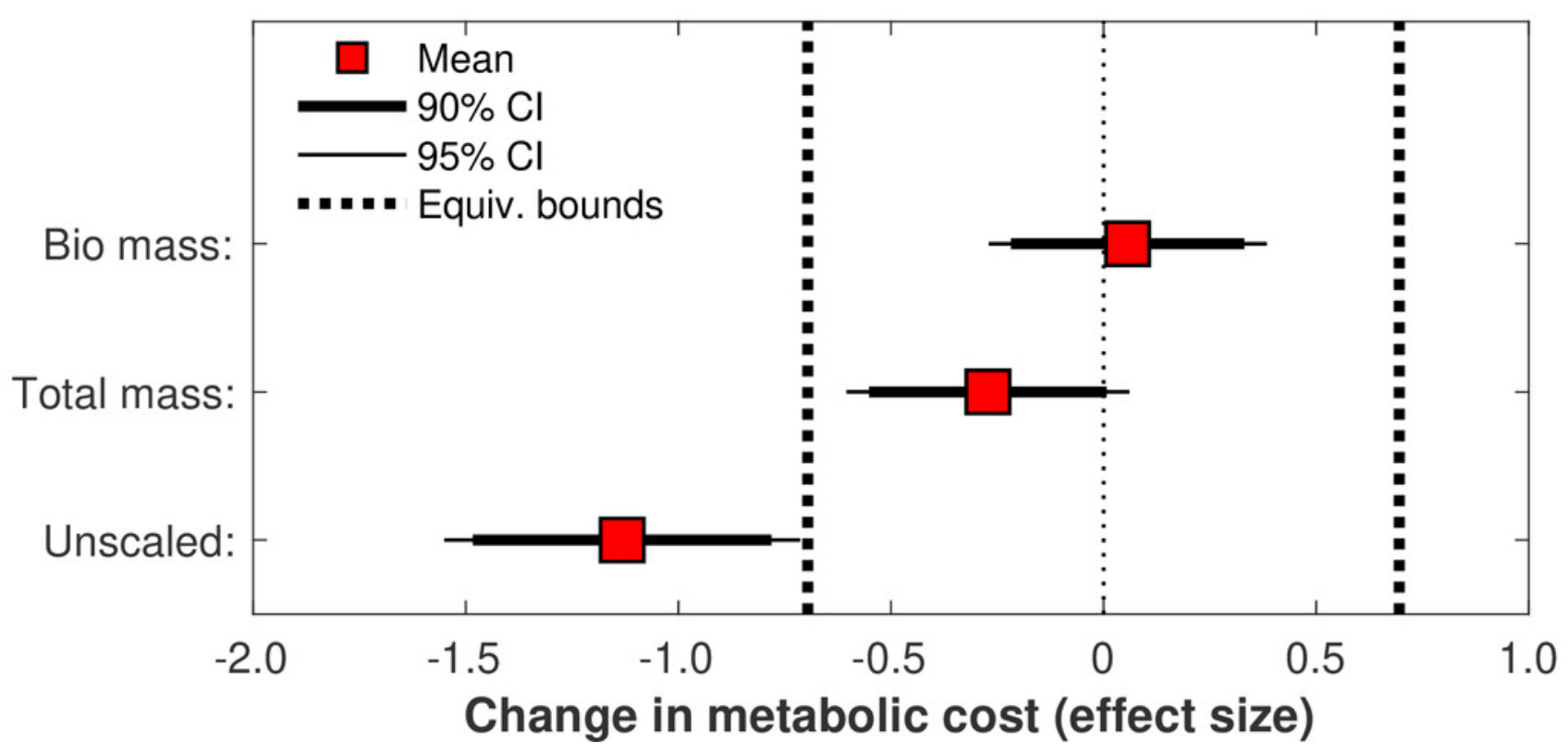




\section{Figure 11}

Energy expended by individual muscles

Metabolic energy expended during the walking stride by each of the model's muscles for the pre-limb loss condition (average of both left and right legs), the post-limb loss intact limb, and the post-limb loss residual limb. Horizontal bar lengths are means over 36 subjects and error bars are standard deviations. Circles are values for Subject \#06, who had the largest increase in metabolic cost post-limb loss. 


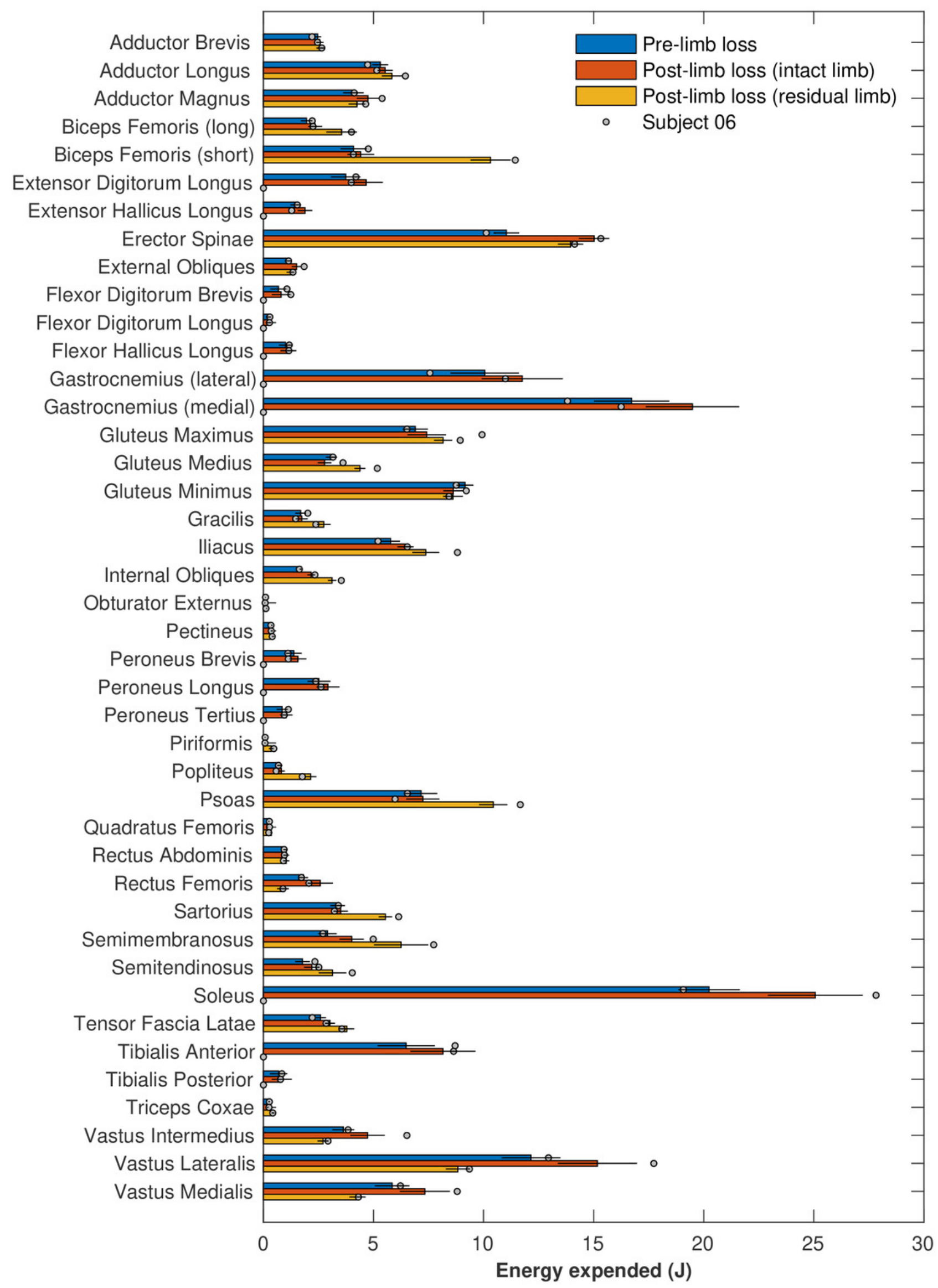




\section{Table $\mathbf{1}$ (on next page)}

Breakdown of energy expenditure

Total metabolic cost per kg biological body mass, and its components. Values of mean \pm standard deviation for the pre- and post-limb loss simulations. The net work and heat sum to the metabolic cost. The concentric and eccentric work sum to the net work. The activation, shortening, and basal heat sum to the net heat. "Whole body" includes all muscles in the model. "Right ankle" includes only the muscles spanning the right ankle in the pre-limb loss model, which were absent in the post-limb loss model. Work-related quantities are the work of muscle fibers, except for the prosthesis, which is the mechanical energy stored/released by the prosthesis. This prosthesis energy does not directly factor into the metabolic cost so is not included under the whole-body energy components. *: The basal heat here is the non-muscular basal heat rate. The basal heat of muscles $(1.0 \mathrm{~W}$ per $\mathrm{kg}$ muscle mass) was included under the muscle activation and shortening heat. The biological body masses were $75.4 \mathrm{~kg}$ pre-limb loss and $72.8 \pm 0.3 \mathrm{~kg}$ post-limb loss. The bottom eight rows present the Joules of energy consumed, unscaled by body mass. 
1

\begin{tabular}{rrrrr} 
& \multicolumn{2}{c}{ Pre-limb loss } & \multicolumn{2}{c}{ Post-limb loss } \\
Quantity (J/m/kg) & Whole body & Right ankle & Whole body & Prosthesis \\
\hline METABOLIC COST: & $3.58 \pm 0.10$ & $0.59 \pm 0.02$ & $3.59 \pm 0.12$ & -- \\
NET WORK: & $0.55 \pm 0.04$ & $0.28 \pm 0.01$ & $0.61 \pm 0.03$ & $-0.01 \pm 0.00$ \\
Concentric: & $1.36 \pm 0.04$ & $0.39 \pm 0.01$ & $1.28 \pm 0.05$ & $0.36 \pm 0.02$ \\
Eccentric: & $-0.81 \pm 0.03$ & $-0.11 \pm 0.01$ & $-0.67 \pm 0.03$ & $-0.37 \pm 0.02$ \\
NET HEAT: & $3.03 \pm 0.08$ & $0.31 \pm 0.01$ & $2.98 \pm 0.09$ & -- \\
Activation: & $2.14 \pm 0.09$ & $0.24 \pm 0.01$ & $2.13 \pm 0.09$ & -- \\
Shortening: & $0.40 \pm 0.01$ & $0.07 \pm 0.01$ & $0.33 \pm 0.02$ & -- \\
*Basal: & $0.49 \pm 0.01$ & -- & $0.51 \pm 0.01$ & - \\
& & & & \\
Quantity (J) & & & & - \\
METABOLIC COST: & $391 \pm 11$ & $65 \pm 2$ & $378 \pm 12$ & - \\
NET WORK: & $60 \pm 5$ & $31 \pm 2$ & $64 \pm 3$ & $-0.5 \pm 0.2$ \\
Concentric: & $149 \pm 5$ & $42 \pm 2$ & $135 \pm 5$ & $26.6 \pm 1.6$ \\
Eccentric: & $-89 \pm 3$ & $-12 \pm 1$ & $-71 \pm 3$ & $-27.1 \pm 1.6$ \\
NET HEAT: & $331 \pm 9$ & $34 \pm 1$ & $314 \pm 10$ & - \\
Activation: & $234 \pm 10$ & $26 \pm 1$ & $225 \pm 10$ & - \\
Shortening: & $44 \pm 2$ & $8 \pm 1$ & $35 \pm 2$ & - \\
*Basal: & $54 \pm 1$ & -- & $54 \pm 1$ & - \\
\hline \hline
\end{tabular}

2 